\title{
Red-Tuning of the Channelrhodopsin Spectrum Using Long Conjugated Retinal Analogues
}

\section{$\operatorname{AUTHOR}(S)$ :}

Shen, Yi-Chung; Sasaki, Toshikazu; Matsuyama, Take; Yamashita, Takahiro; Shichida, Yoshinori; Okitsu, Takashi; Yamano, Yumiko; ... Ishizuka, Toru; Yawo, Hiromu; Imamoto, Yasushi

\section{CITATION:}

Shen, Yi-Chung ... [et al]. Red-Tuning of the Channelrhodopsin Spectrum Using Long Conjugated Retinal Analogues. Biochemistry 2018, 57(38): 5544-5556

\section{ISSUE DATE:}

2018-09-25

URL:

http://hdl.handle.net/2433/243173

\section{RIGHT:}

This document is the Accepted Manuscript version of a Published Work that appeared in final form in Biochemistry, copyright (c) American Chemical Society after peer review and technical editing by the publisher. To access the final edited and published work see https://doi.org/10.1021/acs.biochem.8b00583.; The full-text file will be made open to the public on 28 August 2019 in accordance with publisher's 'Terms and Conditions for Self-Archiving'.; This is not the published version. Please cite only the published version.; この論文は出版社版でありません。引用の際には出版社版 をご確認ご利用ください。 


\title{
Red-Tuning of Channelrhodopsin Spectrum Using Long-Conjugated Retinal Analogues
}

\author{
Yi-Chung Shen, ${ }^{\dagger}$ Toshikazu Sasaki, ${ }^{\dagger}$ Take Matsuyama, ${ }^{\dagger}$ Takahiro Yamashita, ${ }^{\dagger}$ Yoshinori \\ Shichida, ${ }^{\dagger, \ddagger}$ Takashi Okitsu, Yumiko Yamano," Akimori Wada," Toru Ishizuka, ${ }^{\S}$ Hiromu
}

$$
\text { Yawo, }{ }^{\S} \text { Yasushi Imamoto }{ }^{*}+
$$

${ }^{\dagger}$ Department of Biophysics, Graduate School of Science, Kyoto University, Kyoto 606-8502, Japan, ${ }^{\ddagger}$ Research Organization of Science and Technology, Ritsumeikan University, Kusatsu, Shiga 525-8577, Japan, "Laboratory of Organic Chemistry for Life Science, Kobe Pharmaceutical University, Kobe, Hyogo 658-0003, Japan, and ${ }^{\S}$ Department of Developmental Biology and Neuroscience, Graduate School of Life Science, Tohoku University, Sendai, Miyagi 980-8577, Japan 
ABSTRACT: As optogenetic studies become more popular, a demand for red-shifted channelrhodopsin is increasing, because blue-green light is highly scattered or absorbed by animal tissues. In this study, we developed a red-shifted channelrhodopsin by elongating the conjugated double bond system of the native chromophore, all-trans-retinal (ATR1). Analogues of ATR1 and ATR2 (3,4-didehydro-retinal) in which an extra C=C bond is inserted at different positions (C6-C7, C10-C11, and C14-C15 positions) were synthesized, and introduced into a widely used channelrhodopsin variant, C1C2 (a chimeric protein of channelrhodopsin-1 and channelrhodopsin-2 from Chlamydomonas reinhardtii). C1C2 bearing these retinal analogues as chromophores showed broadened absorption spectra toward the long wavelength side, and photocycle intermediates similar to the conducting state of channelrhodopsin. However, the position of methyl groups on the retinal polyene chain influenced the yield of pigment, absorption maximum, and photocycle pattern to a variable degree. The lack of a methyl group at position C9 of the analogues considerably decreased the yield of pigment, whereas a methyl group at position C15 exhibited a large red-shift in the absorption spectra of the C1C2 analogue. Expansion of the chromophore binding pocket by mutation of the aromatic residue Phe265 to Ala improved the yield of pigment bearing elongated ATR1 analogues without great alteration of the photocycle kinetics of C1C2. Our results show that elongation of the conjugated double bond system of retinal is a promising 
strategy to improve the ability of channelrhodopsin to absorb long-wavelength light passing through the biological optical window. 


\section{INTRODUCTION}

Microbial rhodopsins, also known as type-I rhodopsins, comprise a family of seventransmembrane photoreceptor proteins found in prokaryotes and lower eukaryotes. ${ }^{1}$ In their native organisms, microbial rhodopsins function as transmembrane ion transporters (ion pump/channel) or as sensory signal transducers when light energy is absorbed. These lightdriven functions are mediated by the trans-cis photoisomerization of the chromophore, alltrans-retinal, which induces the formation of a series of intermediate states in the photocycle. To this date, numerous microbial rhodopsins have been discovered and characterized by spectrophotometric and physiologic approaches.

Channelrhodopsin (ChR) is a light-gated cation channel found in algae, which serves as receptor for photo-motility..$^{2-4}$ Many ChR variants have been discovered so far, showing various action spectra over a wide wavelength range, from $436 \mathrm{~nm}$ (Tetraselmis striata) to 590 $\mathrm{nm}$ (Chlamydomonas nocitigama), ${ }^{5}$ as well as variety of ion selectivity. Nowadays, ChRs and their modified proteins are widely utilized in optogenetics. ${ }^{6}$ Heterogeneously expressed ChRs on the cell membrane of a target cell can efficiently depolarize the cell in response to light. ${ }^{7-9}$ However, most naturally occurring ChRs have their maximal sensitivity to blue-green light, ${ }^{5}$ which is highly attenuated in animal tissue due to scattering and the absorption of hemoglobin. To overcome the drawbacks of middle-wavelength absorption, development of ChRs absorbing long-wavelength light has become an important goal. 
Methods to develop long-wavelength absorbing channelrhodopsins include modification of the apoprotein by searching for naturally occurring red-shifted variants, site-directed mutagenesis, or both. Gene screening, ${ }^{10,}{ }^{11}$ rational protein mutagenesis, ${ }^{12-14}$ and chimeric protein construction ${ }^{15-17}$ have been performed in previous studies, exploring and constructing several red-shifted ChR variants. However, most of these ChR variants showed limited extent of red-shift.

Substitution of the native chromophore by a chemically modified one is another canonical approach to achieve rhodopsin color-tuning. For example, a previous study has shown great spectral red-shift of proton pump rhodopsins, proteorhodopsin and Gloeobacter rhodopsin, by regenerating the pigments with retinal analogues that have stronger electron-withdrawing groups. ${ }^{18}$ These approaches show that enhancing the conjugation of double bond system of the chromophore is a good strategy to red-shift the ChR spectra. In most cases, both microbial and animal rhodopsins naturally use A1-retinals (retinal-1) as a chromophore. However, some fresh water fish and amphibians use A2-retinals (3,4-didehydro-retinal, retinal-2) as the chromophore in their visual rhodopsin. ${ }^{19-22}$ The additional double bond on the ring of A2retinal shifts the absorption band of these visual pigments towards a longer wavelength region. ${ }^{23}$ In previous studies, all-trans-A2-retinal (ATR2) and/or other ring-modified retinal analogues were incorporated into ion pumps and channel rhodopsins which originally used alltrans-A1-retinal (ATR1) as their chromophore, and formed red-shifted pigments with retained 
pump/channel activity. ${ }^{24-31}$ The general strategy in these studies is to introduce additional double bond(s) into the $\beta$-ionone ring of retinal.

Following this strategy, elongation of the retinal polyene chain to extend the conjugated $\pi$ electron system could be an alternative straightforward method for further red-shifting. In our previous study, long-conjugated ATR analogue with an enamine-type Schiff base in its structure was noncovalently incorporated into a ChR mutant in which the retinal-binding Lys was replaced by Gly, and efficiently red-shifted the absorption spectrum. ${ }^{32}$ However, chainelongated analogues covalently bound to Lys via Schiff base linkage would be more desirable because the protonated Schiff base is the core of the hydrogen-bonding network, which is essential for the stability and light-induced conformational change of retinal proteins. This approach is challenging because the elongated chromophore would induce extensive steric hindrance on the chromophore binding pocket. In fact, previous attempts to introduce chainelongated retinal analogue into bacteriorhodopsin resulted in the loss of photoactivity and limited color tuning. ${ }^{33,34}$ However, insertion of extra double bond into the polyene chain of ATR alters the spatial relationship between methyl groups, $\beta$-ionone ring, and Schiff base. Thus, careful consideration of the position of the extra double bond insertion may improve the efficiency of pigment formation because methyl groups are also involved in key interactions with the chromophore binding pocket. ${ }^{34,35}$ 
In the present study, we synthesized ATR1 and ATR2 analogues, whose polyene chains are elongated by inserting $\mathrm{C}=\mathrm{C}$ at $\mathrm{C} 6-\mathrm{C} 7, \mathrm{C} 10-\mathrm{C} 11$, and $\mathrm{C} 14-\mathrm{C} 15$ positions, herein referred to as ATR1(ATR2)-6ex, ATR1(ATR2)-10ex, and ATR1(ATR2)-14ex, respectively. These analogues were incorporated into the widely used C1C2 ChR variant, ${ }^{36}$ a stable chimeric channelrhodopsin of $\mathrm{ChR}^{37}$ and $\mathrm{ChR}^{38}$, from Chlamydomonas reinhardtii. Our results demonstrate that the absorption spectra of $\mathrm{C} 1 \mathrm{C} 2$ were broadened toward the long wavelength side by both ATR1 and ATR2 analogues. The position of $\mathrm{C}=\mathrm{C}$ insertion on retinal polyene chain influenced the relative yield of purified pigment, absorption maximum, and photocycle kinetics in varying degrees, but a photocycle intermediate, similar to conducting state of C1C2, was formed. Expansion of the chromophore binding pocket by mutation of an aromatic amino acid residue improved the yield of pigment with elongated retinal analogues. Our results show that elongation of the conjugated double bond system of retinal, not only on the $\beta$-ionone ring but also on the polyene chain, is a promising strategy to bathochromically tune channelrhodopsins, which improves their ability to utilize long-wavelength light. 


\section{MATERIALS AND METHODS}

Synthesis of One-Double-Bond-Extended Retinal Analogues. ATR2-6ex, ATR2-10ex, and ATR2-14ex were prepared as previously reported. ${ }^{39}$ ATR1-14ex was prepared from ATR1 by the Horner-Wadsworth-Emmons reaction with C2-phosphonate (triethyl phophonoacetate), and subsequent conversion of ester to aldehyde by lithium aluminum hydride or diisobutyl aluminum hydride reduction and manganese dioxide oxidation. ATR1-10ex was prepared from $\beta$-ionylideneacetaldehyde ${ }^{40}$ by two rounds of the Horner-Wadsworth-Emmons reaction with C2-phosphonate and C5-phosphonate (triethyl (E)-3-methyl-4-phosphonocrotonate) accompanied with functional transformation of ester to aldehyde. ATR1-6ex was prepared from commercially available $\beta$-cyclocitral by three rounds of the Horner-Wadsworth-Emmons reaction with C2-phosphonate, C5-phosphonate, and C5-phosphonate, respectively, accompanied with functional transformation of ester to aldehyde.

Preparation of C1C2 Analogues and Mutants. In this study, C1C2, a stable chimeric channelrhodopsin of ChR1 and ChR2 from Chlamydomonas reinhardtii, which consists of the N-terminal loop and helices 1-5 of ChR1 and the helices 6-7 and C-terminal loop of ChR2, was used as a template. ${ }^{41}$ The cDNA of C1C2 chimera was inserted into the mammalian expression vector pCAGGS ${ }^{42}$ with the epitope sequence of anti-bovine rhodopsin monoclonal antibody rho1D4 (ETSQVAPA) on the C-terminus. The cDNAs of C1C2 containing a point mutation 
(W163A, F217A, W262A, F265A, or F269A) were constructed using the In-Fusion cloning kit

(Clontech) according to the manufacturer's instructions.

For the protein expression, plasmids were transfected into HEK293 cells using the calciumphosphate method. After incubation for 2 days, transfected cells were collected by centrifugation and suspended in Buffer P (50 mM HEPES, 140 mM NaCl, pH 6.5). Collected cells were supplemented with retinal or analogue dissolved in ethanol at final concentration of $20 \mu \mathrm{M}$, and incubated overnight at $4^{\circ} \mathrm{C}$ to form the pigments. The regenerated pigments were extracted by Buffer P supplemented with $1 \% n$-dodecyl- $\beta$-D-maltoside (DDM), and were subjected to rho1D4-antibody conjugated agarose column, to which C-terminal rho1D4 sequence of each protein specifically binds. After extensively washing the column with Buffer P with $0.02 \%$ DDM, the proteins were eluted by adding excess amount of the synthetic peptide corresponding to the epitope sequence of rho1D4. The final solvent of the sample for the following measurements was Buffer P with 0.02\% DDM (0.02\% DDM, 50 mM HEPES, 140 $\mathrm{mM} \mathrm{NaCl}, \mathrm{pH}$ 6.5). All the procedures above were performed under deep-red light. When C1C2 was similarly purified without addition of any retinal or analogues, $\sim 10 \%$ of fully regenerated pigment with excess ATR1 was formed, indicating that the endogenous ATR1 in HEK293 cells regenerates small amounts of pigment. 
Spectroscopy. Absorption spectra of pigments at 250-750 nm were measured by UV-visible spectrophotometer (Shimadzu UV-2400), which is equipped with a water circulation system to the cell holder to keep the sample at $4^{\circ} \mathrm{C}$.

Photoreaction of the pigment was measured by a laboratory-built time-resolved spectrophotometer system. The monitoring light, which was generated by a UV-vis fiber light source (L10290, Hamamatsu Photonics), was passed through the sample cell and focused on a polychromator (SpectraPro-150, Acton Research Corporation). The photoreaction was initiated with a yellow flash (>430 nm, pulse width of $170 \mu$ s) generated by a xenon flash lamp (Nissin Electronic) with a Y-45 glass cutoff filter (AGC Techno Glass). The full spectra (208-930 nm) with a wavelength resolution of $0.52 \mathrm{~nm}$ were recorded by a high-speed time delay integration CCD camera (C10000, Hamamatsu Photonics). The temperature of the sample was kept at $20^{\circ} \mathrm{C}$ by a Peltier-controlled cuvette holder (qpod, Ocean Optics).

In the first $500 \mathrm{~ms}$ after flash excitation, spectra were continuously recorded every $200 \mu \mathrm{s}$, and the spectra at desired time points were obtained by averaging proper number of spectra (e.g. average of 10 spectra for $<10 \mathrm{~ms}$ and 128 for $>100 \mathrm{~ms}$ ). Then averaged spectra of 128 measurements were recorded at desired time points up to 2 min. The sample was kept in the dark before the measurements, and the series of transient spectra obtained by a single flash excitation are presented. 
Data Analysis. All spectroscopic data were analyzed and plotted using the software Igor Pro 6.37 (WaveMetrics Inc.). For the time-resolved analyses of photocycles, the spectral changes were analyzed by singular value decomposition (SVD) method, which was described in our previous study in detail. ${ }^{43,44}$ In short, the datasets of transient difference spectra were arranged in a matrix $\mathbf{A}$, in which the columns and rows correspond to wavelength and time after photoexcitation, respectively. The SVD calculation decomposed A into the product of a left singular matrix $\mathbf{U}$, a diagonal matrix containing singular values $\mathbf{S}$, and a transpose of a right singular matrix $\mathbf{V}$. If the difference in amplitudes between the $i$ th and (i+1)th singular values was significantly greater than that between the $(i+1)$ th and $(i+2)$ th ones, number of significant components ( $n$ ) was determined to be $i$ as follows.

$$
\mathbf{A}=\mathbf{U} \times \mathbf{S} \times \mathbf{V}^{\mathrm{T}} \approx \mathbf{U}_{n} \times \mathbf{S}_{n} \times \mathbf{V}_{n}^{\mathrm{T}}
$$

where $\mathbf{U}_{n}$ and $\mathbf{V}_{n}^{\mathrm{T}}$ contain the first $n$ columns of $\mathbf{U}$ and $\mathbf{V}^{\mathrm{T}}$, and $\mathbf{S}_{n}$ contains the first $n$ singular values. To determine the time constant (reciprocal value of rate constant) of each transition, columns of $\mathbf{V}_{n}^{\mathrm{T}}$ were global-fitted by the sum of exponential functions as follows:

$$
\mathbf{V}_{n}^{\mathrm{T}} \approx \mathbf{C}_{n} \times\left(1, \exp \left(-t / \tau_{1}\right), \exp \left(-t / \tau_{2}\right), \cdots, \exp \left(-t / \tau_{n-1}\right)^{\mathrm{T}}\right.
$$

From Eqs. 1 and 2,

$$
\mathbf{A} \approx \mathbf{U}_{n} \times \mathbf{S}_{n} \times \mathbf{C}_{n} \times\left(1, \exp \left(-t / \tau_{1}\right), \exp \left(-t / \tau_{2}\right), \cdots, \exp \left(-t / \tau_{n-1}\right)\right.
$$


Columns of $\mathbf{U}_{n} \times \mathbf{S}_{n} \times \mathbf{C}_{n}$ are the decay-associated difference spectra (B-spectra). The transient difference spectra at time $=t(\operatorname{TDS}(t))$ were approximated by the combination of Bspectra as follows.

$$
\operatorname{TDS}(t)=\mathbf{B}_{0}+\mathbf{B}_{1} \exp \left(-t / \tau_{1}\right)+\mathbf{B}_{2} \exp \left(-t / \tau_{2}\right)+\cdots+\mathbf{B}_{n-1} \exp \left(-t / \tau_{n-1}\right)
$$

where $\mathbf{B}_{i}$ are B-spectra, and $\tau_{i}$ are the time constants. Therefore, the extrapolated difference spectra at $t=0$ and $t=\infty$ (DS 0 and $\mathbf{D S}_{\infty}$, respectively), and those of pseudo-steady state after the $i$ th transition (DSi) were calculated as follows:

$$
\left\{\begin{array}{l}
\mathbf{D S}_{0}=\mathbf{B}_{0}+\mathbf{B}_{1}+\mathbf{B}_{2}+\cdots+\mathbf{B}_{n-1}=\mathbf{T D S}(0) \\
\mathbf{D S}_{1}=\mathbf{D S}_{0}-\mathbf{B}_{1} \\
\mathbf{D S}_{2}=\mathbf{D S}_{1}-\mathbf{B}_{2} \\
\quad \vdots \\
\mathbf{D S}_{n-1}=\mathbf{D S}_{\infty}=\mathbf{B}_{0}=\mathbf{T D S}(\infty)
\end{array}\right.
$$

By estimating the fraction of photoexcited pigment ( $f$ ), the absorption spectra (AS) at $t=0, t$ $=\infty$, and in the pseudo-steady state, were calculated as follows:

$$
\mathbf{A S}_{i}=\mathbf{D S}_{i}+f \times \mathbf{D A R K}
$$

where DARK is the dark-state spectra. $f$ was estimated so that all $\mathbf{A} \mathbf{S}_{i}$ have no negative absorbance. Note that $\mathbf{A S}_{i}$ possibly represents the mixture of intermediates. 


\section{RESULTS}

Absorption Characteristics of Retinal and Analogues. Retinal and its analogues used in the current experiments are listed in Figure 1 and Table 1. We used two naturally existing retinals, all-trans-retinal (ATR1) and 3,4-didehydro-all-trans-retinal (ATR2), and their $\mathrm{C}=\mathrm{C}$ elongated analogues on positions C6-C7 (ATR1-6ex and ATR2-6ex), C10-C11 (ATR1-10ex and ATR2-10ex), and C14-C15 (ATR1-14ex and ATR2-14ex). The absorption spectra of these retinal and analogues are shown in Figure 1i, and the absorption characteristics are listed in Table 1. It is well known that the absorption spectrum of ATR2 is 15-20 nm red-shifted from ATR1 due to the additional double bond in $\beta$-ionone ring. Elongation of a conjugated double bond system by inserting $\mathrm{C}=\mathrm{C}$ in its polyene chain red-shifted the absorption maximum by approximately 15-20 nm for both ATR1 and ATR2 (Table 1). Note that ATR2, ATR1-6ex, ATR1-10ex, and ATR1-14ex showed similar absorption spectra, while those of ATR2-6ex, ATR2-10ex, and ATR2-14ex are similar. In solution, the absorption spectra of retinal and analogues depends solely on the length of the conjugated double bond system. 
(a) ATR1<smiles>CC1=C(/C=C/C(C)=C/C=C/C(C)=C/C=O)C(C)(Br)CCC1</smiles>

(c) ATR1-6ex<smiles>CC1=C(/C=C/C=C(C)/C=C/C=C/C(C)=C/C=O)C(C)(C)CCC1</smiles>

(e) ATR1-10ex<smiles>CC1=C(/C=C/C(C)=C/C=C/C=C/C(C)=C/C=O)C(C)(Br)CCC1</smiles>

(g) ATR1-14ex<smiles>CC1=C(/C=C/C(C)=C/C=C/C(C)=C/C=C/C=O)C(C)(C)CCC1</smiles>

(b) ATR2<smiles></smiles>

(d) ATR2-6ex<smiles>CC1=C(/C=C/C=C/C(C)=C/C=C/C(C)=C/C=O)C(C)(C)CC=C1</smiles>

(f) ATR2-10ex<smiles>CCC=C/C(C)=C(C)\C=C\C=C\C=C\C=C\C(C)=C\C=O</smiles>

(h) ATR2-14ex

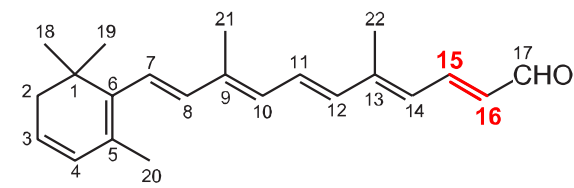

(i)

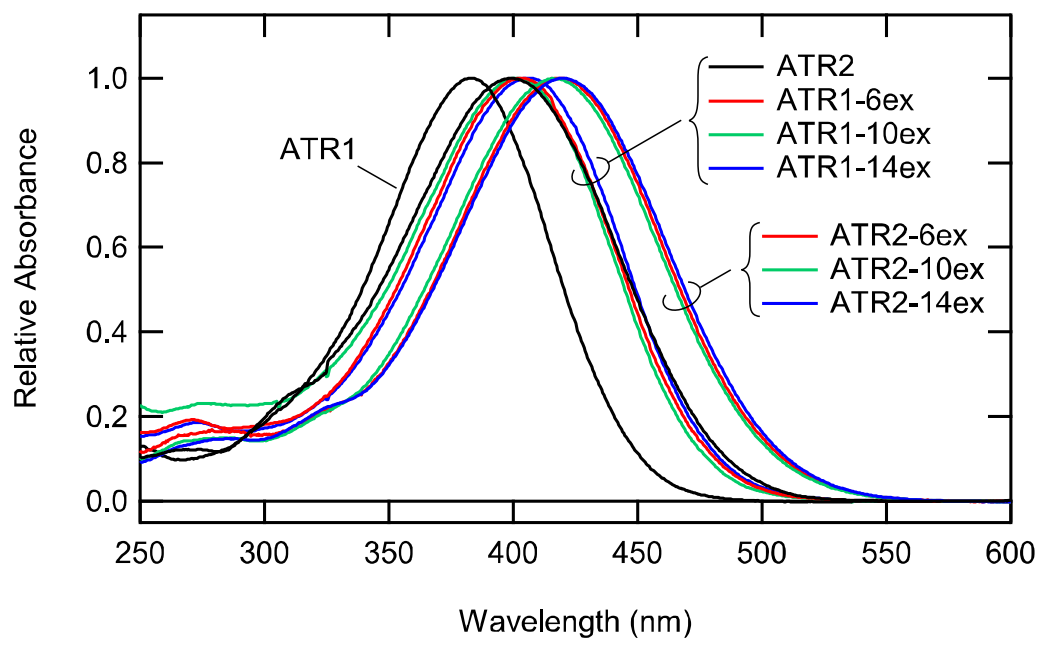

Figure 1. Structures and UV-visible absorption spectra of ATR1, ATR2, and their analogues. (a) ATR1. (b) ATR2. (c) ATR1-6ex. (d) ATR2-6ex. (e) ATR1-10ex. (f) ATR2-10ex. (g) ATR1-14ex. (h) ATR2-14ex. Atoms in red numbers represent the insertion position of $\mathrm{C}=\mathrm{C}$ for each analogue. (i) UV-visible absorption spectra in ethanol. All the absorption spectra were normalized to 1.0. 
Table 1. Absorption characteristics of ATR1, ATR2 and their analogues in ethanol.

\begin{tabular}{|c|c|c|c|c|c|c|c|c|}
\hline & \multicolumn{4}{|c|}{ ATR1- type } & \multicolumn{4}{|c|}{ ATR2- type } \\
\hline & ATR1 & $6 e x$ & $10 \mathrm{ex}$ & $14 \mathrm{ex}$ & ATR2 & $6 e x$ & $10 \mathrm{ex}$ & $14 \mathrm{ex}$ \\
\hline$\lambda_{\max }(\mathrm{nm})$ & 383 & 402 & 401 & 405 & 400 & 418 & 416 & 420 \\
\hline $\operatorname{shift}(\Delta \mathrm{nm})$ & - & 19 & 18 & 22 & 17 & 35 & 33 & 37 \\
\hline
\end{tabular}

Absorption Characteristics of C1C2 with Retinal Analogues. Unlike extended retinal analogues in solution, the absorption spectra of C1C2 bearing these retinal analogues as the chromophore varied widely (Figure 2 and Table 2). C1C2 with the native chromophore, ATR1 (ATR1-C1C2), shows a maximum absorbance at $477 \mathrm{~nm}$, with two characteristic shoulders at approximately $450 \mathrm{~nm}$ and $415 \mathrm{~nm}$. The long-wavelength spectral boundary, at which absorbance is $1 / e$ of maximal absorbance, ${ }^{24}$ is $515 \mathrm{~nm}$ (Table 2). Using ATR2 as a chromophore (ATR2-C1C2), the absorption maximum and long-wavelength spectral boundary of C1C2 sample was red-shifted by 7 and $27 \mathrm{~nm}$, respectively (Table 2). In addition, the spectral shoulders observed in ATR1-C1C2 were less prominent in ATR2-C1C2. Because small amount of ATR1-C1C2 generated with endogenous ATR1 in HEK293 expression system causes the blue-shift of the absorption spectrum of the sample, absorption maximum and longwavelength spectral boundary of pure ATR2-C1C2 would be more red-shifted. 


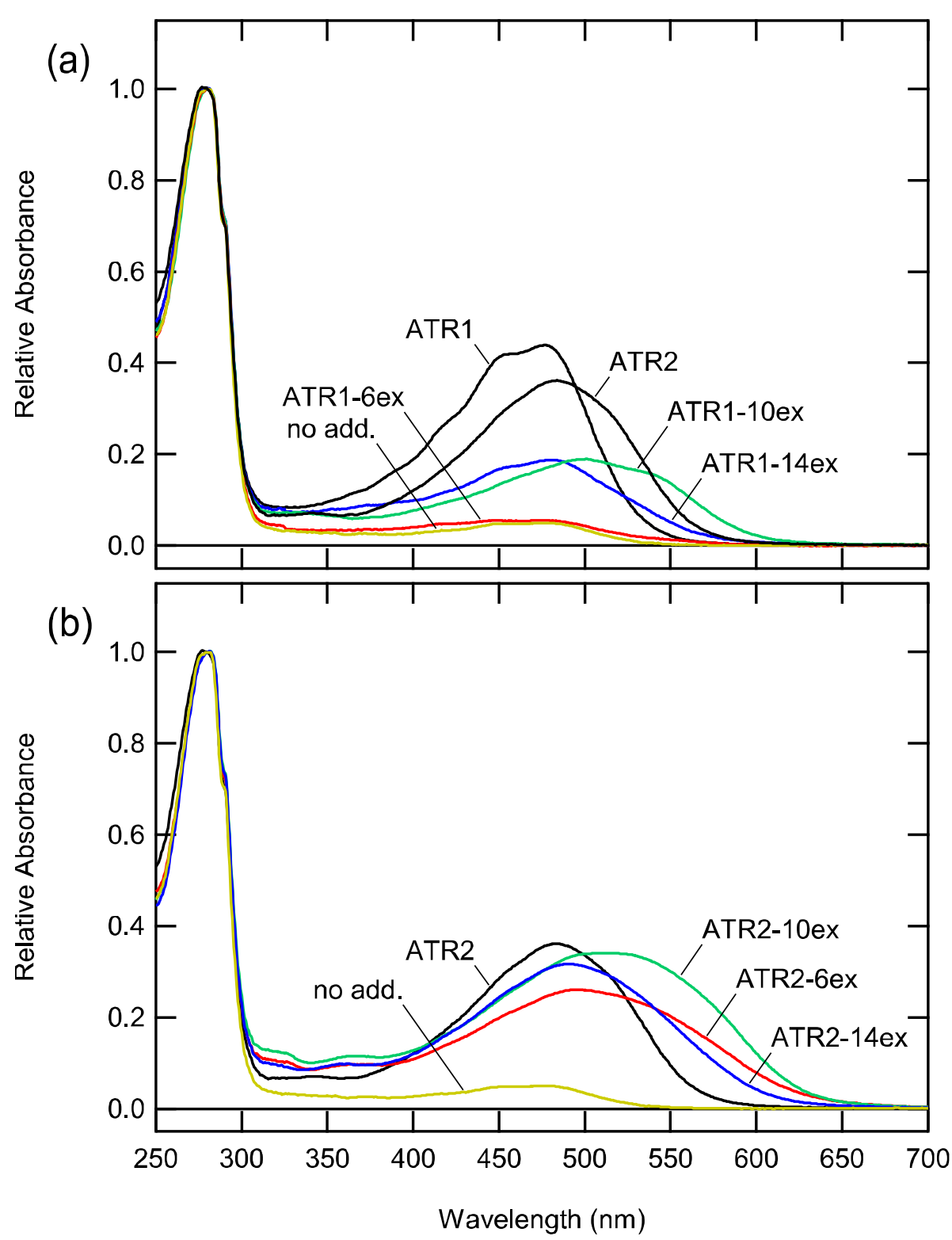

Figure 2. UV-visible absorption spectra of C1C2 bearing ATR1 or its analogues (a) and ATR2 or its analogues (b). All spectra were measured in buffer (0.02\% DDM, 50 mM HEPES, 140 $\mathrm{mM} \mathrm{NaCl}, \mathrm{pH}$ 6.5), and normalized at $278 \mathrm{~nm}$, so that absorbance equals to 1 . A small amount of ATR1 pigment was formed without addition of retinal and analogues (no add.). 
Table 2. Absorption characteristics of C1C2 analogues.

\begin{tabular}{|c|c|c|c|c|c|c|c|c|}
\hline & \multicolumn{4}{|c|}{ ATR1- type } & \multicolumn{4}{|c|}{ ATR2- type } \\
\hline & ATR1 & $6 e x$ & $10 \mathrm{ex}$ & $14 \mathrm{ex}$ & ATR2 & $6 e x$ & $10 \mathrm{ex}$ & $14 \mathrm{ex}$ \\
\hline$\lambda_{\max }(\mathrm{nm})$ & 477 & - & 502 & 479 & 484 & 493 & 510 & 490 \\
\hline shift $(\Delta \mathrm{nm})$ & - & - & 25 & 2 & 7 & 16 & 33 & 13 \\
\hline Long-WL boundary (nm) & 515 & - & 574 & 540 & 542 & 593 & 599 & 572 \\
\hline $\operatorname{shift}(\Delta \mathrm{nm})$ & - & - & 59 & 25 & 27 & 78 & 84 & 57 \\
\hline Relative absorbance ${ }^{\mathrm{b}}$ & $\begin{array}{c}0.431 \\
\pm 0.008\end{array}$ & $\begin{array}{c}0.075 \\
\pm 0.011\end{array}$ & $\begin{array}{c}0.182 \\
\pm 0.013\end{array}$ & $\begin{array}{c}0.178 \\
\pm 0.015\end{array}$ & $\begin{array}{c}0.360 \\
\pm 0.007\end{array}$ & $\begin{array}{c}0.250 \\
\pm 0.013\end{array}$ & $\begin{array}{c}0.328 \\
\pm 0.015\end{array}$ & $\begin{array}{c}0.299 \\
\pm 0.019\end{array}$ \\
\hline
\end{tabular}

Values were obtained from the absorption spectra in buffer (0.02\% DDM, 50 mM HEPES, 140 $\mathrm{mM} \mathrm{NaCl}, \mathrm{pH}$ 6.5). Because the blue-shift of the apparent absorption spectra by the contribution of endogenous ATR1 pigment is not taken into consideration, the values of pure analogue pigments are possibly more red-shifted. ${ }^{\text {a }}$ The wavelength at which the absorbance is 1/e of maximal absorbance. ${ }^{\mathrm{b}}$ The value of $\mathrm{Abs}_{\max } / \mathrm{Abs}_{278}$. Each value is the mean \pm standard deviation of 3-4 independent measurements.

Replacing the chromophore from ATR1 and ATR2 with their elongated analogues tunes the absorption maximum and the long-wavelength spectral boundary to varying degrees (Figure 2 and Table 2). ATR1-6ex-C1C2 showed almost no absorption peak, indicating that the chromophore binding pocket of C1C2 could not efficiently accommodate ATR1-6ex as its chromophore. ATR1-10ex red-shifted the absorption spectrum of C1C2 most effectively between three elongated ATR1 analogues, with 25-nm red-shifted absorption maximum, and the two spectral shoulders were observed at approximately 455 and $545 \mathrm{~nm}$. The longwavelength spectral boundary of ATR1-10ex-C1C2 was also remarkably extended to $574 \mathrm{~nm}$ (Table 2). ATR1-14ex only 2-nm red-shifted the absorption maximum of C1C2, with retained 
spectral shoulder at approximately $450 \mathrm{~nm}$. However, the long-wavelength spectral boundary of ATR1-14ex-C1C2 was efficiently extended to $540 \mathrm{~nm}$ (Table 2). Note that the length of conjugated double bond system of these elongated ATR1 analogues is the same as that of ATR2, while the absorption maxima, as well as the long-wavelength boundaries of these C1C2 analogues varied widely.

When elongated ATR2 analogues were incorporated into C1C2, the absorption spectra were further red-shifted as compared to their corresponding ATR1 analogues (Figure 2). The absorption maxima of ATR2-6ex-C1C2, ATR2-10ex-C1C2, and ATR2-14ex-C1C2 were redshifted by 16, 33, and $13 \mathrm{~nm}$, respectively, from that of ATR1-C1C2 (Table 2). The spectral shoulder was less prominent in C1C2 bearing ATR2 analogues than in those bearing ATR1 analogues, and long-wavelength spectral boundaries of ATR2-6ex-C1C2, ATR2-10ex-C1C2, and ATR2-14ex-C1C2 were extended to 593 nm, 599 nm, and 572 nm, respectively (Table 2). Again, note that trace amounts of endogenous ATR1 from HEK293 expression system could generate pigment with C1C2 (Figure 2), which possibly blue-shifts the absorption spectra of the sample. The absorption maxima and long-wavelength boundaries of analogue pigments presented here are possibly underestimated, because the contribution of ATR1 pigment was not taken into consideration. The expanded long wavelength spectral boundaries would allow these pigments to respond to light from Nd-YAG laser (532 nm), green He-Ne laser (543 nm), and red He-Ne laser (633 nm) 5-20 times more than original ATR1-C1C2. 
The relative yield of pigment with these retinal analogues was assessed by the relative absorbance, which is the maximal absorbance in visible region divided by absorbance at 278 nm (Abs max $_{\text {Abs278) }}$ (Table 2). While relative absorbance depends on the binding affinity of analogues to the chromophore binding site, it would be affected by the difference in the extinction coefficients and stability of formed pigments as well. Absmax/Abs278 of all the ATR1 analogues incorporated into C1C2 were relatively low, whereas those of elongated ATR2 analogues were greater than those of their ATR1 counterparts. For instance, Absmax/Abs278 of ATR2-6ex-C1C2 was better than that of all ATR1-C1C2 analogues, while negligible amount

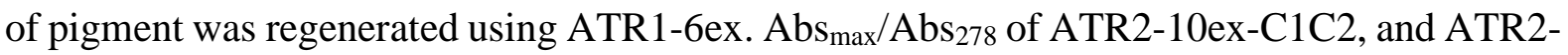
14ex-C1C2 were comparable to that of ATR2-C1C2.

Photoreaction of C1C2 with Retinal Analogues. Previous studies have shown that the chemical modification of retinal chromophore may affect not only the absorption characteristics but also the photocycle kinetics of microbial rhodopsin, such as the altered intermediate states, accelerated/decelerated transitions, and even loss of photoactivity. ${ }^{45,46}$ In this study, the elongated polyene chain and/or the methyl group at altered location could also cause a steric effect. Because the ion channel of ChRs transiently opens during photocycle, it is of importance to assess the alteration of photocycle by chromophore modification. 
Flash-induced spectral changes of C1C2 bearing retinal and analogues were measured by time-resolved UV-visible spectroscopy (Figure S1). Measurements are presented as contour plots, where absorbance increase, and decrease, are shown in red/yellow, and cyan/blue, respectively (Figure 3). So far, the photocycle of channelrhodopsin has been extensively investigated using C. reinhardtii ChR2, where the serial formation of intermediate states, $\mathrm{P} 1^{500}$, $\mathrm{P}^{390}, \mathrm{P}^{520}$, and $\mathrm{P} 4^{480}$, were reported, ${ }^{47}$ among which $\mathrm{P}^{520}$ and possibly $\mathrm{P}{ }^{390}$ are considered to be conducting states. Although the photocycle of C1C2 is still not fully understood, a previous report suggested that it is similar to that of ChR2. ${ }^{48}$ 

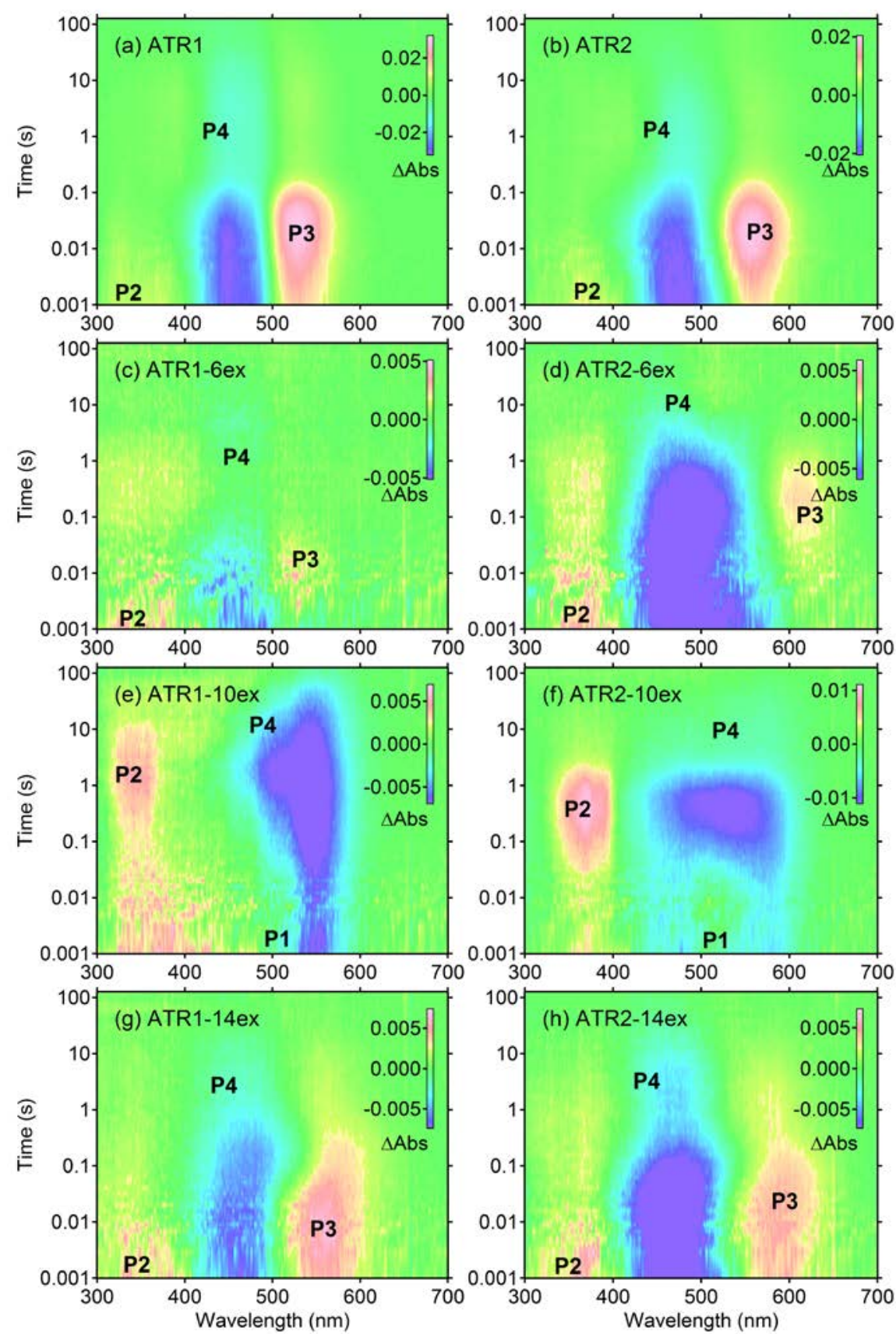

Figure 3. Time-resolved UV-visible absorption spectroscopy of C1C2 bearing ATR1, ATR2, or their analogues. Contour plots were generated by Igor Pro. Absorbance increase and decrease are shown in red/yellow and cyan/blue, respectively. Original transient spectra obtained by excitation with a yellow flash $(>430 \mathrm{~nm}$, pulse duration $=170 \mu \mathrm{s})$ are shown in Figure S1. All measurements were performed in buffer (0.02\% DDM, 50 mM HEPES, $140 \mathrm{mM} \mathrm{NaCl}$, pH 6.5) at $20^{\circ} \mathrm{C}$. (a) ATR1-C1C2. (b) ATR2-C1C2. (c) ATR1-6ex-C1C2. (d) ATR2-6ex-C1C2. (e) ATR1-10ex-C1C2. (f) ATR2-10ex-C1C2. (g) ATR1-14ex-C1C2. (h) ATR2-14ex-C1C2. 
When ATR1-C1C2 was excited by a yellow flash, the absorbance decreased at $470 \mathrm{~nm}$ and increased at $350 \mathrm{~nm}$ and $530 \mathrm{~nm}$ as observed right after flash (Figures 3a and S1a). Then, absorbance at $350 \mathrm{~nm}$ decreased with concomitant increase at $530 \mathrm{~nm}$, followed by absorbance decrease at $530 \mathrm{~nm}$, while small loss of absorbance at $450 \mathrm{~nm}$ was long-lived in the photocycle. To further anatomize the spectral changes in our measurements, the series of transient difference spectra (Figure S1a) were analyzed by singular value decomposition (SVD) analysis, ${ }^{43,}{ }^{44}$ giving four significant U- and V-spectra. By global fitting of four V-spectra with three-exponential function, three decay associated difference spectra $\left(\mathbf{B}_{1}, \mathbf{B}_{2}\right.$, and $\left.\mathbf{B}_{3}\right)$ plus constant component (B) were calculated (Eq. 4) (Figure 4a). $\mathbf{B}_{1}-\mathbf{B}_{3}$, oppositely signed for easy comparison in Figure 4, represent the spectral changes (different spectra) of respective transition between intermediate states, which have their individual time constant $(\tau)$. For comparison, extrapolated difference spectrum at $t=0\left(\mathbf{D S}_{0}\right)(\mathrm{Eq} .5)$ is also presented in Figure 4a. 

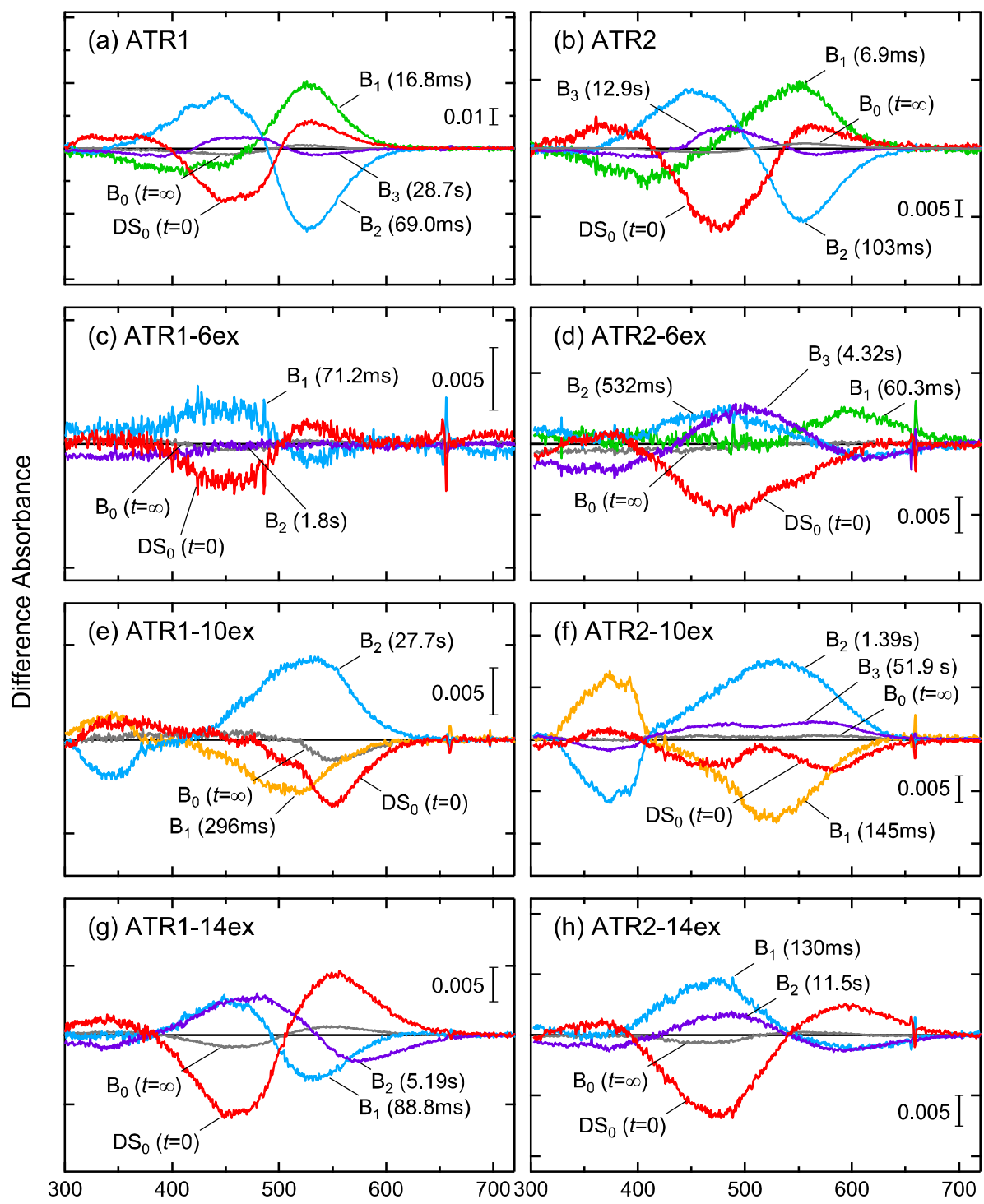

Wavelength $(\mathrm{nm})$

(h) ATR2-14ex

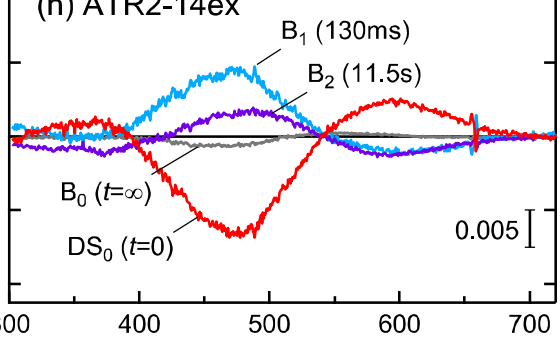

Figure 4. SVD analyzed time-resolved UV-visible absorption spectra of C1C2 bearing ATR1, ATR2, or their analogues. DS $_{0}$ (red) represents the extrapolated difference spectra at $t=0$ (Eq. 5). The decay-associated difference spectra $\left(\mathbf{B}_{1}, \mathbf{B}_{2}\right.$, and $\left.\mathbf{B}_{3}\right)$ are oppositely signed for easy comparison. Orange, green, blue, and purple lines tentatively represent the formations of $\mathrm{P}^{390}$, $\mathrm{P}^{520}, \mathrm{P}^{480}$, and dark state, respectively. $\mathbf{B}_{0}$, (gray) represents the extrapolated difference spectra at $t=\infty$ (Eqs. 4 and 5). All measurements were performed with a yellow flash (>430 $\mathrm{nm}$, pulse duration $=170 \mu \mathrm{s})$ in buffer $(0.02 \%$ DDM, $50 \mathrm{mM}$ HEPES, $140 \mathrm{mM} \mathrm{NaCl}, \mathrm{pH}$ 6.5) at $20^{\circ} \mathrm{C}$. (a) ATR1-C1C2. (b) ATR2-C1C2. (c) ATR1-6ex-C1C2. (d) ATR2-6ex-C1C2. (e) ATR1-10ex-C1C2. (f) ATR2-10ex-C1C2. (g) ATR1-14ex-C1C2. (h) ATR2-14ex-C1C2. Note that the spectral changes for ATR1-10ex (e) and ATR2-10ex (f) are quite different from the others (for detail, see text). 
To better understand the spectral changes, extrapolated absorption spectra at $t=0\left(\mathbf{A S}_{0}\right)$ and $t=\infty\left(\mathbf{A} \mathbf{S}_{\infty}\right)$, and pseudo steady states $\left(\mathbf{A S}_{1}, \mathbf{A S}_{2}\right.$, and $\left.\mathbf{A} \mathbf{S}_{3}\right)$ were generated using $\mathbf{B}_{0}-\mathbf{B}_{3}$ spectra and dark-state spectrum of ATR1-C1C2 (Eq. 6) (Figure S2). SVD-analyzed spectra of ATR1C1C2 (Figures 4a and S2a) imply that the absorbance of dark-state was significantly decreased and the mixture of $\mathrm{P} 2^{390}$ and $\mathrm{P}^{520}$ (and possibly $\mathrm{P} 1^{500}$ ) was formed just after the flash photoexcitation ( $\mathbf{D S}_{0}$ and $\left.\mathbf{A S}_{0}\right) . \mathbf{B}_{1}(\tau=17 \mathrm{~ms})$ corresponded to the conversion from $\mathrm{P} 2^{390}$ to $\mathrm{P}^{520}$ (green), and gave the extrapolated absorption spectra mainly containing $\mathrm{P}^{520}\left(\mathbf{A S}_{1}\right)$. Similarly, B2 ( $\tau=69 \mathrm{~ms}$ ), corresponding to the conversion from $\mathrm{P} 3^{520}$ to $\mathrm{P} 4^{480}$ (blue), gave the spectra mainly containing P4 ${ }^{480}\left(\mathbf{A S}_{2}\right)$. B3 ( $\tau=29$ s) represents the recovery of the dark-state by the decay of the final intermediate ( $\mathrm{P} 4^{480}$ ) (purple), and $\mathbf{B}_{0}$ and $\mathbf{A} \mathbf{S}_{\infty}$ were almost identical to base line and the dark-state spectrum, respectively.

For the analyses of the flash-induced spectral changes of C1C2 analogues, B spectra, and resulted DS and AS were calculated as well. The photocycle of ATR2-C1C2 (Figures 3b, 4b, S1b and S2b) were qualitatively in good agreement with that of ATR1-C1C2, while the spectra were somewhat red-shifted, implying that the addition of double bond on $\beta$-ionone ring did not largely affect the photocycle kinetics of C1C2. On the other hand, addition of double bond on polyene chain shows various effects on the photocycle of C1C2 (Figures 3c-h, 4c-h, S1c-h, and S2c-h). ATR1-6ex-C1C2 (Figures 4c and S2c), which formed almost no pigment, showed a negligible photo-reaction signal, which could be attributed to a minute amount of ATR1-C1C2 
formed by endogenous ATR1 in HEK cells. Meanwhile, C1C2 with other analogues showed photo-reaction with detectable intermediates by light activation. ATR2-6ex-C1C2 (Figures 3d, 4d, S1d, and S2d), ATR1-14ex-C1C2 (Figures 3g, 4g, S1g, and S2g) and ATR2-14ex-C1C2 (Figures 3h, 4h, S1h, and S2h) showed a photocycle similar to ATR1-C1C2, in which the formation of UV-intermediate ( $\mathrm{P} 2^{390}$ : hereafter absorption maxima of corresponding intermediates of ATR1-C1C2 are indicated by suffixes for easy comparison) just after flash excitation, and red-shifted intermediate $\left(\mathrm{P}^{520}\right.$ ) were formed (Figures 3d, 3g, and 3h). However, time constants and absorption maxima of these intermediates were slightly changed, e.g., the formation of $\mathrm{P}^{520}$ was decelerated in ATR2-6ex-C1C2, while P3 ${ }^{520}$ of ATR1-14ex-C1C2 and ATR2-14ex-C1C2 were formed within the present time resolution $(<2 \mathrm{~ms}$, note that green curve showing $\mathrm{P}^{520}$ formation is missing in Figures $4 \mathrm{~g}$ and $4 \mathrm{~h}$. See also Figures S2g and S2h). In contrast, the spectral changes for ATR1-10ex-C1C2 (Figures 3e, 4e, S1e, and S2e) and ATR2-10ex-C1C2 (Figures 3f, 4f, S1f, and S2f) appeared to be quite different from those of ATR1-C1C2. Two intermediates could be clearly detected: one, formed just after flash excitation, had the absorption spectrum similar to that of dark-state, and the other was UVintermediate (Figures 3e, 3f, S2e, and S2f). These two intermediates would thus be corresponded to $\mathrm{P} 1^{500}$ and $\mathrm{P} 2^{390}$, respectively, while their decays were extremely decelerated. The following red-shifted intermediate, $\mathrm{P} 3^{520}$, was not clearly observed. However, small loss of absorbance at approximately $500 \mathrm{~nm}$ was long-lived, indicating the formation of $\mathrm{P} 4^{480}$. The 
decay of $\mathrm{P} 4^{480}$ of ATR1-C1C2-10ex was so slow that $\mathrm{P} 4^{480}$ decay was not observed in the present 130 s measurement (Figures 3e, 4e, and S2e), while ATR2-C1C2-10ex showed P4480 decay time constant of $51.9 \mathrm{~s}$ (Figures 3f, 4f, and S2f). It is likely that $\mathrm{P3}^{520}$ could not be observed in ATR1-C1C2-10ex and ATR2-C1C2-10ex because the lifetime of $\mathrm{P}^{390}$ was significantly longer than that of $\mathrm{P} 3^{520}$.

According to the results of time-resolved UV-visible spectroscopy and SVD analysis, the photocycle of C1C2 bearing elongated retinal analogues is consistently explained by the intermediates known in ATR1-C1C2 photocycle, but the kinetics are substantially altered.

Modification of Chromophore Binding Pocket. The pigment formation of elongated ATR1 analogue was poorer than that of ATR1, as suggested by their significantly lower relative absorbances (Abs $\max / \mathrm{Abs}_{278}$ ) compared to those of ATR1 and ATR2 (Table 2). It is likely that the elongated retinal is not properly accommodated in the chromophore binding pocket due to the steric hindrance. This may become an issue when introducing these elongated retinal analogues in optogenetic studies, as effective pigments would decrease. Therefore, to eliminate the extra steric hindrance caused by elongated retinal analogues, the bulky aromatic amino acid side chain located near the retinal chromophore was removed by site directed mutagenesis. 


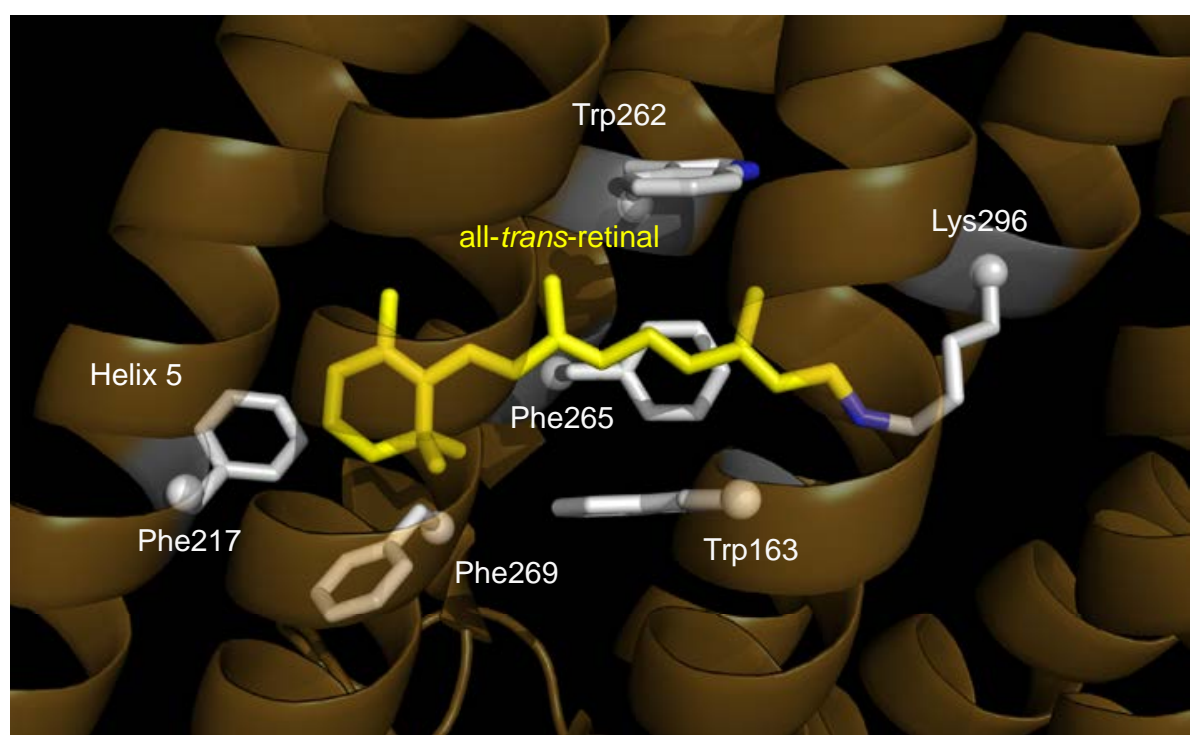

Figure 5. Structure of chromophore binding pocket in C1C2 (PDB: 3ug9). Retinal forms Schiff base with Lys296, and is surrounded by five aromatic amino acids, Trp163, Phe217, Trp262, Phe265, and Phe269. Phe217 and Phe269 locate near the $\beta$-ionone ring, while the rest 3 residues locate near the polyene chain of retinal.

Crystal structure of $\mathrm{C} 1 \mathrm{C} 2^{36}$ demonstrated that the chromophore binding pocket is composed of 5 aromatic amino acid residues, Trp163, Phe217, Trp262, Phe265, and Phe269 (Figure 5). We mutated each residue to alanine (W163A, F217A, W262A, F265A, and F269A), and assessed the relative yield of pigment by ATR1 and ATR1 analogues (Figure S3). All mutants yielded the pigment with ATR1, while the expression levels were somehow different. However, elongated retinal analogues could only be incorporated into F265A to form pigments. Thus, the pigment formation yield of F265A were further tested in detail, with both ATRs and ATR analogues (Figure 6 and Table 3). Comparing to C1C2, F265A mutation caused minor tuning to the absorption maxima, but the long-wavelength boundaries of ATR1-F265A and ATR2F265A were 12-15 nm red-shifted. The yields of pigments of F265A with ATRs and analogues 
were then judged from the relative absorbances (Absmax/Abs278) (Figures 6b). F265A showed a modestly reduced Absmax/Abs278 with ATR1, but significantly increased Absmax/Abs278 with ATR1 analogues, especially with ATR1-10ex and ATR1-14ex (Figure 6c). However, Abs $_{\max } / \mathrm{Abs}_{278}$ of ATR2 analogues did not evidently improved by F265A mutation. That is probably because ATR2 analogues already have near-saturated regeneration efficiency to C1C2 (Figure 6c).

Table 3. Absorption characteristics of C1C2-F265A analogues.

\begin{tabular}{|c|c|c|c|c|c|c|c|c|}
\hline & \multicolumn{4}{|c|}{ ATR1- type } & \multicolumn{4}{|c|}{ ATR2- type } \\
\hline & ATR1 & $6 e x$ & $10 \mathrm{ex}$ & $14 \mathrm{ex}$ & ATR2 & $6 e x$ & $10 \mathrm{ex}$ & $14 \mathrm{ex}$ \\
\hline$\lambda_{\max }(\mathrm{nm})$ & 476 & 483 & 501 & 475 & 494 & 496 & 529 & 486 \\
\hline $\operatorname{shift}(\Delta \mathrm{nm})$ & - & 7 & 25 & -1 & 18 & 20 & 53 & 10 \\
\hline Long-WL boundary (nm) & 527 & 556 & 573 & 532 & 557 & 602 & 601 & 561 \\
\hline shift $(\Delta \mathrm{nm})$ & - & 29 & 46 & 5 & 30 & 75 & 74 & 34 \\
\hline Relative absorbance $^{\mathrm{b}}$ & $\begin{array}{c}0.407 \\
\pm 0.007\end{array}$ & $\begin{array}{c}0.081 \\
\pm 0.009\end{array}$ & $\begin{array}{c}0.250 \\
\pm 0.049\end{array}$ & $\begin{array}{r}0.306 \\
\pm 0.032\end{array}$ & $\begin{array}{c}0.388 \\
\pm 0.018\end{array}$ & $\begin{array}{c}0.128 \\
\pm 0.003\end{array}$ & $\begin{array}{c}0.322 \\
\pm 0.005\end{array}$ & $\begin{array}{r}0.302 \\
\pm 0.005\end{array}$ \\
\hline
\end{tabular}

Values were obtained from the absorption spectra in buffer (0.02\% DDM, 50 mM HEPES, 140 $\mathrm{mM} \mathrm{NaCl}, \mathrm{pH}$ 6.5). Because the blue-shift of the apparent absorption spectra by the contribution of endogenous ATR1 pigment is not taken into consideration, the values of pure analogue pigments are possibly more red-shifted. ${ }^{\text {a }}$ The wavelength at which the absorbance is 1/e of maximal absorbance. ${ }^{\mathrm{b}}$ The value of $\mathrm{Abs}_{\max } / \mathrm{Abs}_{278}$. Each value is the mean \pm standard deviation of 3-4 independent measurements. 

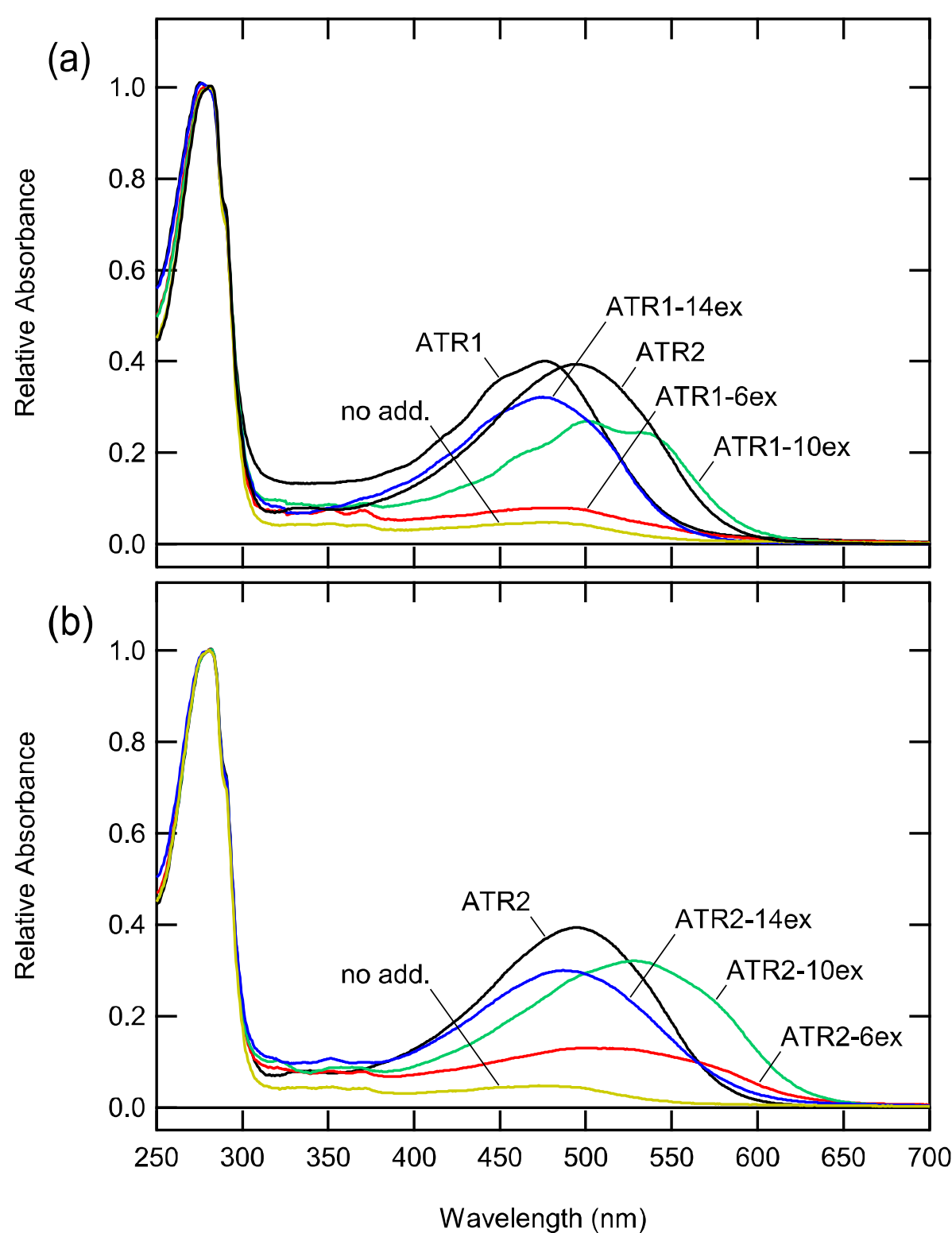

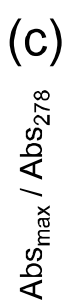

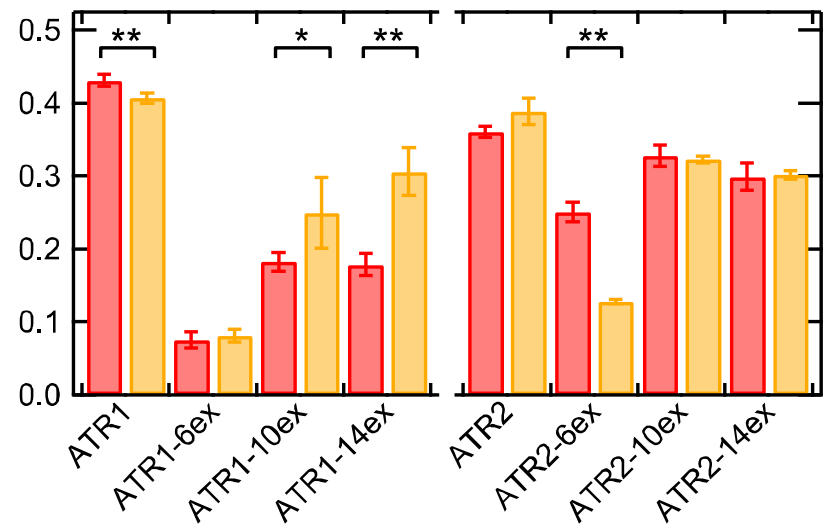

Chromophore

Figure 6. UV-visible absorption spectra of F265A bearing ATR1 or their analogues (a) and ATR2 or its analogues (b). All spectra were measured in buffer (0.02\% DDM, 50 mM HEPES, 
$140 \mathrm{mM} \mathrm{NaCl}, \mathrm{pH}$ 6.5), and normalized at $278 \mathrm{~nm}$, so that absorbance equals to 1 . (c) Comparison of the yields of pigment formation between C1C2 (red) and F265A (orange) by relative absorbances ( $\mathrm{Abs}_{\max } / \mathrm{Abs}_{278}$ ). Values are means of 3-4 independent measurements and the error bars show the standard deviations. ${ }^{*} p<0.02$, ${ }^{* *} p<0.002$.

The photocycle of F265A bearing ATRs and analogues were also analyzed with timeresolved spectroscopy (Figures 7 and S4) and SVD analysis (Figures 8 and S5). The results demonstrated that the photocycle intermediates are qualitatively similar to C1C2 counterparts, while the time constants for transition were altered. For instance, P3 ${ }^{520}$ formation in ATR1F265A photocycle (Figure 8a, S5a) was significantly accelerated ( $\tau=<2 \mathrm{~ms}$ ) as compared to that of ATR1-C1C2 (17 ms). However, the P4 ${ }^{480}$ formation was decelerated from 69 to $471 \mathrm{~ms}$, and the recovery to dark-state was decelerated from 28 to $87 \mathrm{~s}$. Similar alterations of photocycle kinetics by F265A mutation such as accelerated formation of $\mathrm{P}^{520}$ and following slowed process, were also observed for other retinal and analogues (Figures 8 and S5). As the channelopen state in the photocycle could be assigned to $\mathrm{P}^{520}$ and potentially $\mathrm{P} 2^{390}$, the slower conversion from $\mathrm{P}^{520}$ to $\mathrm{P} 4^{480}$ caused by F265A suggests a prolonged channel-open duration per photocycle. Interestingly, ATR1-6ex-F265A, whose yield of pigment formation was poor similarly to ATR1-6ex-C1C2, showed a photocycle signal distinguishable from ATR1-F265A, which was considered as originated by ATR1-6ex analogue. These results again prove that the structure of channelrhodopsin, which binds elongated retinal analogues, was stabilized by F265A mutation. 

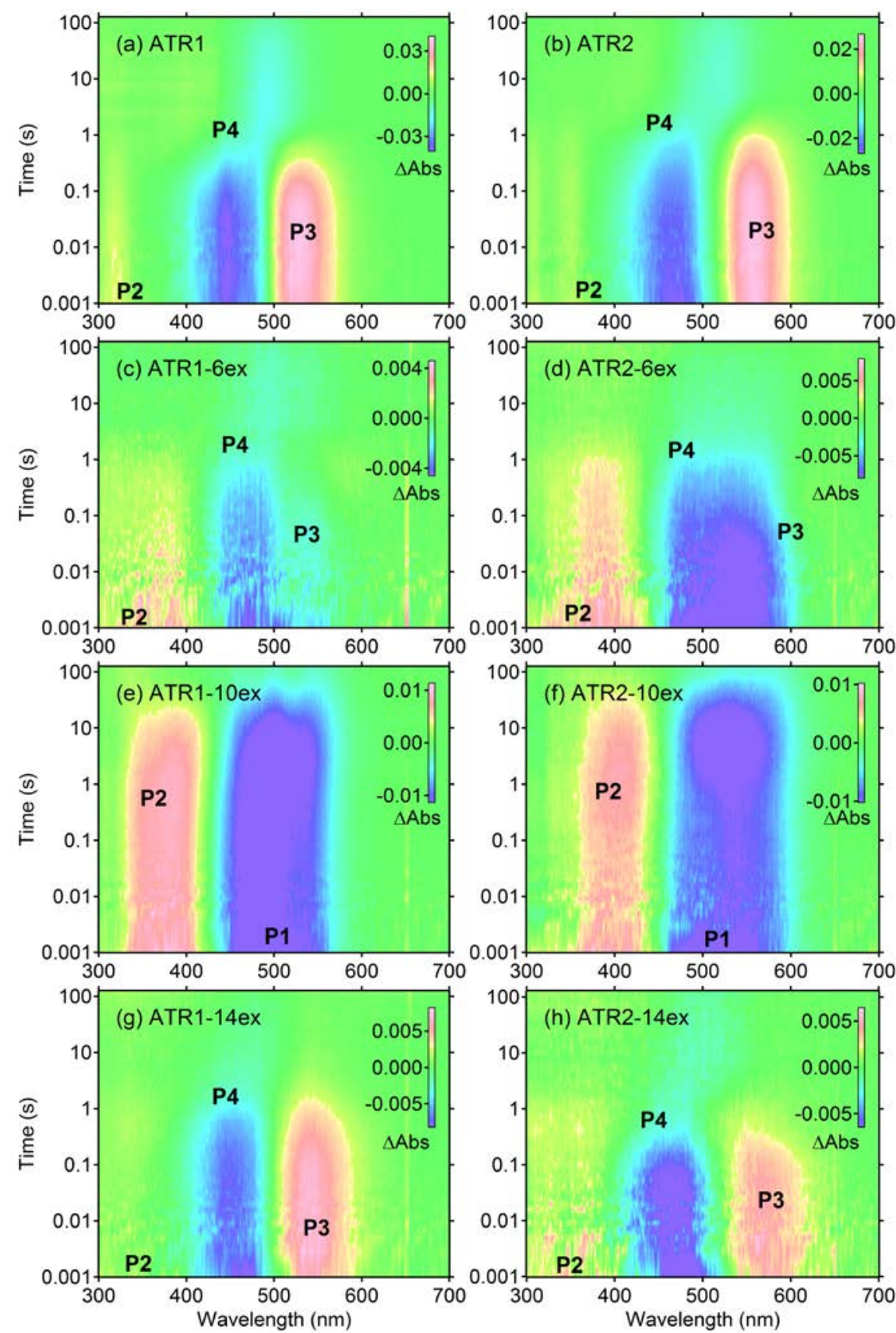

Figure 7. Time-resolved UV-visible absorption spectroscopy of C1C2-F265A bearing ATR1, ATR2, or their analogues. Contour plots were generated by Igor Pro. Absorbance increase and decrease are shown in red/yellow and cyan/blue, respectively. Original transient spectra obtained by excitation with a yellow flash $(>430 \mathrm{~nm}$, pulse duration $=170 \mu \mathrm{s})$ are shown in Figure S4. All measurements were performed in buffer (0.02\% DDM, $50 \mathrm{mM}$ HEPES, 140 $\mathrm{mM} \mathrm{NaCl}, \mathrm{pH} 6.5$ ) at $20^{\circ} \mathrm{C}$. (a) ATR1-F265A. (b) ATR2-F265A. (c) ATR1-6ex-F265A. (d) ATR2-6ex-F265A. (e) ATR1-10ex-F265A. (f) ATR2-10ex-F265A. (g) ATR1-14ex-F265A. (h) ATR2-14ex-F265A. 

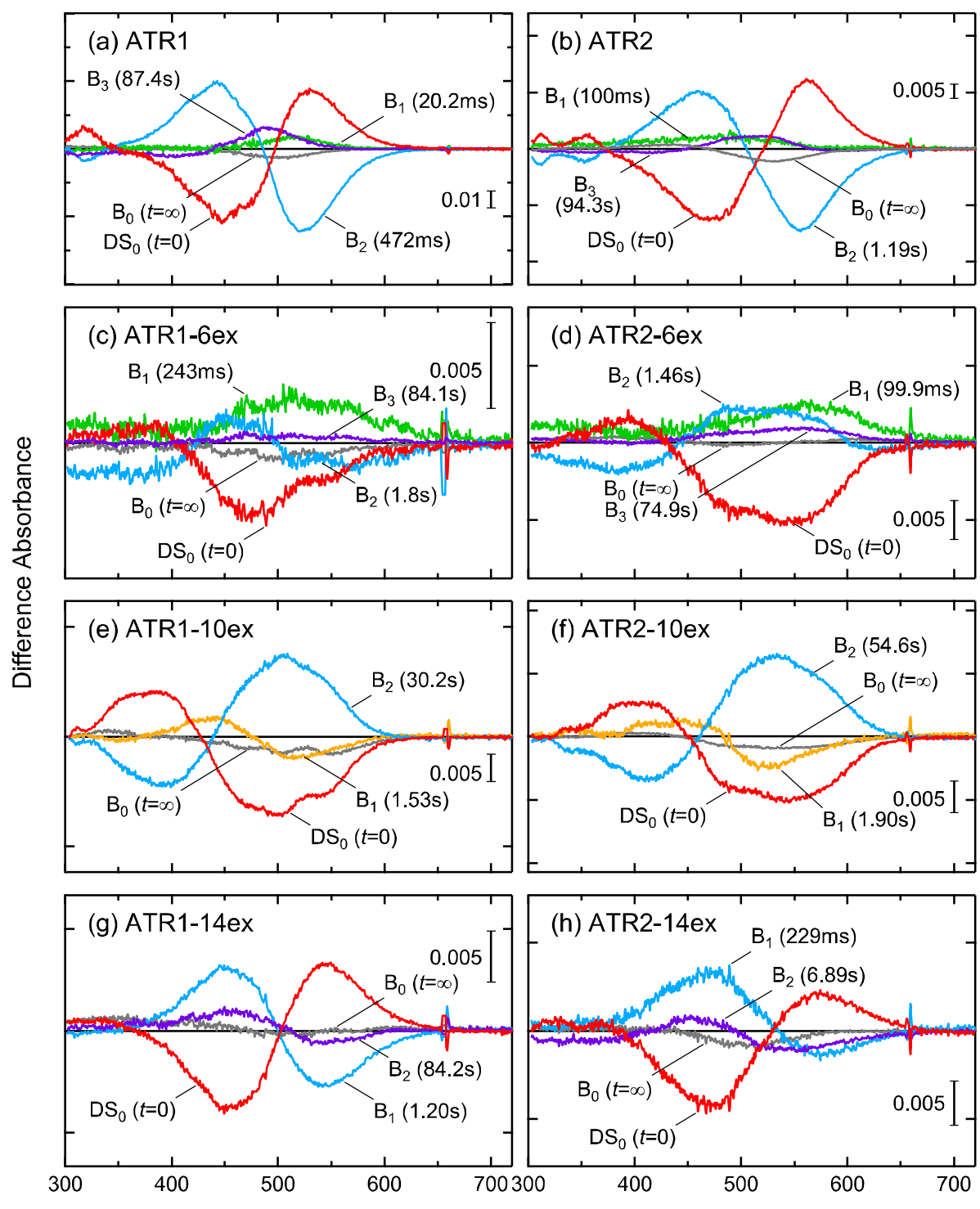

Wavelength $(\mathrm{nm})$

Figure 8. SVD-analyzed time-resolved UV-visible absorption spectra of F265A bearing ATR1, ATR2, or their analogues. DS $_{0}$ (red) represents the extrapolated difference spectra at $t=0$ (Eq. 5). The decay-associated difference spectra $\left(\mathbf{B}_{1}, \mathbf{B}_{2}\right.$, and $\left.\mathbf{B}_{3}\right)$ are oppositely signed for easy comparison. Orange, green, blue, and purple lines tentatively represent the formations of $\mathrm{P} 2^{390}$, $\mathrm{P}^{520}, \mathrm{P} 4{ }^{480}$, and dark state, respectively. $\mathbf{B}_{0}$, (gray) represents the extrapolated difference spectra at $t=\infty$ (Eqs. 4 and 5). All measurements were performed with a yellow flash (> 430 $\mathrm{nm}$, pulse duration=170 $\mu \mathrm{s})$ in buffer (0.02\% DDM, $50 \mathrm{mM}$ HEPES, $140 \mathrm{mM} \mathrm{NaCl}, \mathrm{pH} 6.5)$ at $20^{\circ} \mathrm{C}$. (a) ATR1-F265A. (b) ATR2-F265A. (c) ATR1-6ex-F265A. (d) ATR2-6ex-F265A. (e) ATR1-10ex-F265A. (f) ATR2-10ex-F265A. (g) ATR1-14ex-F265A. (h) ATR2-14ex-F265A. 


\section{DISCUSSION}

In this study, we demonstrated that the color-tuning of ChRs could be achieved by adding additional conjugated double bond(s), not only on the $\beta$-ionone ring, ${ }^{24}$ but also on its polyene chain. Although the red-shift in maximal absorption wavelength is relatively small, the absorption spectrum was effectively broadened, resulting in a significant red-shift of the longwavelength boundary. Free ATR analogues having one additional double bond (ATR1-6ex, ATR1-10ex, ATR1-14ex, and ATR2) showed equally red-shifted absorption spectra relative to that of ATR1 (Figure 1i, Table 1). Two additional double bonds (ATR2-6ex, ATR2-10ex, and ATR2-14ex) further red-shifted the absorption spectra, and their absorption spectra were comparable (Figure 1i, Table 1). These findings indicate that the absorption spectra of free ATR in solution solely depends on the length of the conjugated double bond system. In contrast, binding experiments with C1C2 demonstrated that the spectral red shift highly depends on the location of the double bond insertion, indicating that the interaction between the chromophore binding pocket and methyl groups in polyene chain of analogues regulates the absorption spectra of C1C2 (Figure 2, Table 2). It should be noted that inserting double bonds in $\beta$-ionone ring and polyene chain additively red-shifted the absorption spectra, and ATR2-6ex-C1C2 and ATR2-10ex-C1C2 could serve as better candidates for optogenetic utilization.

However, the elongation of polyene chain raises an issue regarding the altered chromophore structure, which may cause extra steric effects and hinder the interaction between the binding 
pocket and chromophore. A previous study ${ }^{34}$ has shown that the incorporation of ATR1-14ex into bleached bacteriorhodopsin purple membrane in place of ATR1 yields unstable pigment which shows no photoactivity. In contrast, our results showed that C1C2 could generate pigment with all ATR1 and ATR2 analogues except for ATR1-6ex (Figure 2, Table 2), and underwent the photocycle which is required for the gating of channel (Figures 3 and 4). Especially, the formations of near-UV intermediate $\left(\mathrm{P} 2^{390}\right)$ and subsequent red-shifted intermediate $\left(\mathrm{P}^{520}\right)$ are notable, since they correspond to the channel-open state.

Elongated Retinal Analogues in the Chromophore Binding Pocket. Elongated retinal analogues may not be perfectly fitted into the C1C2 chromophore binding pocket, which has limited space. Our mutagenesis analysis gives an insight into how these elongated analogues could be accommodated in the chromophore binding pocket (Figures 5 and S3). Mutation of the aromatic residues near retinal $\beta$-ionone ring, Phe217 and Phe269, resulted in no pigment formation with elongated ATR1 analogues. This indicates that the hydrophobic interaction between $\beta$-ionone ring and these two residues, which holds the $\beta$-ionone ring of elongated analogues in its native position, is essential for forming pigments. In fact, the crystal structure of C1C2 suggests that linearly extended all-trans structure of elongated retinal analogues is hindered by the steric interaction between $\beta$-ionone ring and helix 5 (Figure 5). However, because the manipulation in binding experiment was carried under the dim red light and the reaction mixture was kept in the dark, it is likely that the double bonds of elongated retinal 
analogues are kept in trans configuration. Assuming that the positions of $\beta$-ionone ring and Schiff base are not largely altered in the elongated chromophore, s-cis structure(s) would be adopted in the polyene chain, bending the structure and shortening the longitudinal distance between $\beta$-ionone ring and Schiff base. The steric hindrance around polyene chain would be thus enhanced, but it could be released by F265A mutation near the chromophore polyene, resulting in the improved yield of pigment. It is likely that the polyene chain was bending toward a vertical direction of polyene plane of the chromophore where Phe265 is located (Figure 5). Two other aromatic residues, Trp163 and Trp262, are located in the extracellular and cytoplasmic side of chromophore binding pocket, respectively. While W163A and W262A mutations appear to release the steric interaction between bent retinal analogue and the chromophore binding pocket, it is not the case, as these mutants generated no pigments with analogues. Therefore, these two residues could be considered to be involved in the hydrophobic packing, which stabilizes the chromophore binding pocket.

The Effect of F265A Mutation to Photochemical Properties of C1C2. The introduction of F265A mutation not only enhanced the Absmax/Abs278 with ATR1-14ex and ATR1-10ex significantly, but also slightly decreased the Abs max/Abs278 with native ATR1 (Figure 6). This is an important property for optogenetic application, since most living cells have endogenous ATR1, which would be readily incorporated into the chromophore binding pocket, competing with the exogenous retinal analogues, and diminishing the effective pigments toward long- 
wavelength light. Similar effect were also found for ATR1-6ex. While photocycle of ATR16ex-C1C2 sample was likely to be originated from endogenous ATR1, ATR1-6ex-F265A showed a photocycle distinct from ATR1-C1C2. F265A mutation favored ATR1-6ex binding, and a distinctive photocycle originated from ATR1-6ex was observed (Figure 7c, 8c). Although the observed effects were modest, our approach would improve the affinity of elongated analogues to the chromophore binding pocket relative to native ATR1.

F265A mutation also affected the photocycle kinetics. So far, studies toward photocycle kinetics of ChR were mainly focused on the hydrophilic residues near the chromophore binding pocket, such as counter ion, ${ }^{12,13}$ DC (Asp-Cys) pair, ${ }^{49-51}$ or O-H cluster, ${ }^{52}$ which are involved in the hydrogen bonding network. Hydrophobic residues are not thought to be directly involved in the proton transfer during the photocycle, but can be structurally essential. Our results showed that the conversion from $\mathrm{P} 2^{390}$ to $\mathrm{P} 3^{520}$ was accelerated, and $\mathrm{P} 3^{520}$ to $\mathrm{P} 4^{480}$ was decelerated by F265A mutation (Figures 3a and 7a). The $\mathrm{P} 2^{390}$ to $\mathrm{P}^{520}$ conversion corresponds to the proton transfer from the proton donor residue, Asp195, to Schiff base, and $\mathrm{P}^{520}$ to $\mathrm{P} 4^{480}$ conversion corresponds to reprotonation of Asp195. ${ }^{53}$ While Phe265 alters deprotonation and reprotonation kinetics of Asp195, Phe265 is located on the opposite side of Asp195 across the chromophore. Therefore, it is not likely that Phe265 directly perturbs the proton transfer pathway. Rather, removal of Phe265 would induce an unfavorable charge environment for proton to approach Asp195 in the chromophore binding pocket, resulting in accelerated proton 
donation and decelerated reprotonation. Accordingly, our result of photocycle kinetics alteration by the F265A mutation gave a new point of view toward engineering of retinalbinding proteins.

The Effect of Different Methyl Position of Analogues on Pigment Photochemical Properties. Another issue is how the position of methyl groups of elongated ATR1 and ATR2 analogues influences the photochemical properties. Among respective series of analogues, the backbone of conjugated double bond system is common, but the positions of methyl groups varied. Unlike in solution, these analogues in the chromophore binding pocket showed strikingly different characteristics from each other: the relative yield of pigment and spectral shift were dependent on the position of methyl group (Table 4). ATR1-14ex-C1C2 and ATR214ex-C1C2 showed the least red-shift of both absorption maximum and long-wavelength boundary, indicating that lack of methyl group at C15 position would lower the electron withdraw ability. On the other hand, ATR1-6ex-C1C2 and ATR2-6ex-C1C2 showed a lower yield of pigment than their 10ex and 14ex counterparts, suggesting that 9-methyl group would be essential for pigment formation and/or the stability of formed pigment. While almost no pigment was formed with ATR1-6ex, ATR2-6ex effectively formed pigment with C1C2, suggesting that the interaction between 9-methyl group and chromophore binding site is compensated by the extra $\pi$-electrons in the $\beta$-ionone ring region. Note that not only ATR26ex but also ATR2-10ex and ATR2-14ex had higher yield of pigment than their ATR1 
counterparts. The extra $\pi$-electrons on the $\beta$-ionone ring of ATR2 would enhance the $\pi$ interaction with the phenyl group(s) of adjacent Phe217 and/or Phe269.

Table 4. Effect of position of methyl groups on the spectroscopic characteristics of C1C2.

\begin{tabular}{|c|c|c|c|c|c|c|c|c|}
\hline & \multicolumn{4}{|c|}{ ATR1- type } & \multicolumn{4}{|c|}{ ATR2- type } \\
\hline & ATR1 & $6 e x$ & $10 \mathrm{ex}$ & $14 \mathrm{ex}$ & ATR2 & $6 e x$ & $10 \mathrm{ex}$ & $14 \mathrm{ex}$ \\
\hline Relative yield of pigment & S & $\mathrm{N}$ & W & $\mathrm{W}$ & $S$ & M & $S$ & $S$ \\
\hline$\lambda_{\max }$ shift & - & - & M & $\mathrm{N}$ & W & M & $\mathrm{S}$ & $\mathrm{W}$ \\
\hline Long-WL boundary shift & - & - & $\mathrm{M}$ & W & W & $S$ & $S$ & $\mathrm{M}$ \\
\hline Photocycle alteration & - & - & $\begin{array}{c}\text { Slow } \\
\mathrm{P} 1^{500}, \mathrm{P}^{390} \\
\text { formation }\end{array}$ & $\begin{array}{c}\text { Fast } \\
\mathrm{P3}^{520} \\
\text { formation }\end{array}$ & $\begin{array}{c}\text { No } \\
\text { Alteration }\end{array}$ & $\begin{array}{c}\text { Slow } \\
\text { P3 }^{520} \\
\text { formation }\end{array}$ & $\begin{array}{c}\text { Slow } \\
\mathrm{P} 1^{500}, \mathrm{P} 2^{390} \\
\text { formation }\end{array}$ & $\begin{array}{c}\text { Fast } \\
\mathrm{P}^{520} \\
\text { formation }\end{array}$ \\
\hline
\end{tabular}

W: weak effect, M: medium effect, S: strong effect, N: nearly no effect, -: control or not observed.

C1C2 bearing these retinal analogues showed substantial differences in the kinetics of the photocycle (Figures 3 and 4, Table 4). ATR2-6ex-C1C2 showed 10 times slower P2 ${ }^{390}$ to P3 320 conversion than that of ATR2-C1C2. Conversely, P2 ${ }^{390}$ to $\mathrm{P}^{520}$ conversion of ATR1-14exC1C2 and ATR2-14ex-C1C2 was completed within detection limit ( $2 \mathrm{~ms}$ ). Since the P2 ${ }^{390}$ to P3 ${ }^{520}$ conversion corresponds to the reprotonation of chromophore Schiff base, 15-methyl group would hinder the approach of proton to deprotonated Schiff base. Unlike 6ex and 14ex analogues, 10ex-C1C2 showed quite different photocycle kinetics from ATR1-C1C2. Although the possibility that the photocycle of 10ex-C1C2 is distinct from that of ATR1-C1C2 
cannot be excluded, it can be consistently explained by extremely decelerated kinetics. $\mathrm{P} 2^{390}$ existed in 100 ms time scale, and $\mathrm{P} 1^{500}$, which should be originally appear in picosecond time scale, could be observed in ms time scale. While $\mathrm{P} 3^{520}$ could not be observed, $\mathrm{P} 4^{480}$ was detected at the end of the reaction. It is likely that the lifetime of $\mathrm{P} 2^{390}$ became significantly longer than that of $\mathrm{P}^{520}$, and the formation of $\mathrm{P} 3^{520}$ was masked by $\mathrm{P} 2^{390}$. Thus, although the photocycle of ATR1- and ATR2-10ex-C1C2 is seemingly quite different from that of ATR1C1C2, it is likely that the events that take place during the photocycle are similar.

To improve this system, further structural details between the chromophore binding pocket and retinal analogues would be needed in the future. Mutagenesis studies and simulation studies for rational design of chromophore binding pocket should be considered. Optimizing the binding between the protein and the retinal analogues leads to more efficient red-shifted pigments, which could be introduced into optogenetic applications. While there is still room for improvement, our approach by elongation of conjugated double bond system is a promising strategy to bathochromically tune channelrhodopsins, to give them the ability to absorb red light. 


\section{ASSOCIATED CONTENT}

\section{Supporting Information}

The Supporting Information is available free of charge on the ACS Publications website.

Time-resolved UV-visible difference absorption spectra, SVD-analyzed absolute spectra of intermediates, UV-visible absorption spectra of C1C2 mutants, and transition time constants of photocycle intermediates (PDF).

\section{AUTHOR INFORMATION}

\section{Corresponding Author}

*Address: Department of Biophysics, Graduate School of Science, Kyoto University, Kyoto 606-8502, Japan. E-mail: imamoto@rh.biophys.kyoto-u.ac.jp.

\section{Author Contributions}

Y. S., A. W. and Y. I. designed the research. T. O., Y. Y., and A. W. prepared long-conjugated retinal analogues. YC. S., T. S., T. M., Y. T., T. I., and H. Y. prepared channelrhodopsin sample. YC. S., T. S., and Y. I. performed the spectroscopic measurements and kinetic analysis. YC. S., A. W., and Y. I. wrote the manuscript with contributions from all other authors.

\section{Funding}

This work was supported by JSPS KAKENHI Grant Numbers JP16K07319 and JP16H02515. 


\section{Notes}

The authors declare no competing financial interest.

\section{ACKNOWLEDGEMENTS}

We are grateful to Prof. Robert S. Molday of the University of British Columbia for providing the hybridoma that produces rho1D4 antibody.

\section{ABBREVIATIONS}

ChR = channelrhodopsin; $\mathrm{DDM}=n$-dodecyl- $\beta$-D-maltoside; ATR1 = all-trans-retinal; ATR2 = 3,4-didehydro-all-trans-retinal; SVD = singular value decomposition; 


\section{REFERENCES}

[1] Ernst, O. P., Lodowski, D. T., Elstner, M., Hegemann, P., Brown, L. S., and Kandori, H. (2014) Microbial and animal rhodopsins: structures, functions, and molecular mechanisms, Chem. Rev. 114, 126-163.

[2] Sineshchekov, O. A., Jung, K. H., and Spudich, J. L. (2002) Two rhodopsins mediate phototaxis to low- and high-intensity light in Chlamydomonas reinhardtii, Proc. Natl. Acad. Sci. U. S. A. 99, 8689-8694.

[3] Govorunova, E. G., Jung, K. H., Sineshchekov, O. A., and Spudich, J. L. (2004) Chlamydomonas sensory rhodopsins A and B: cellular content and role in photophobic responses, Biophys. J. 86, 2342-2349.

[4] Berthold, P., Tsunoda, S. P., Ernst, O. P., Mages, W., Gradmann, D., and Hegemann, P. (2008) Channelrhodopsin-1 initiates phototaxis and photophobic responses in chlamydomonas by immediate light-induced depolarization, Plant Cell 20, 1665-1677.

[5] Schneider, F., Grimm, C., and Hegemann, P. (2015) Biophysics of channelrhodopsin, Annu. Rev. Biophys. 44, 167-186.

[6] Staff, N. (2010) Insights of the decade. Stepping away from the trees for a look at the forest. Introduction, Science 330, 1612-1613.

[7] Govorunova, E. G., and Koppel, L. A. (2016) The road to optogenetics: microbial rhodopsins, Biochemistry (Mosc.) 81, 928-940.

[8] Boyden, E. S. (2015) Optogenetics and the future of neuroscience, Nat. Neurosci. 18, 12001201.

[9] Deisseroth, K. (2015) Optogenetics: 10 years of microbial opsins in neuroscience, Nat. Neurosci. 18, 1213-1225.

[10] Zhang, F., Prigge, M., Beyrière, F., Tsunoda, S. P., Mattis, J., Yizhar, O., Hegemann, P., and Deisseroth, K. (2008) Red-shifted optogenetic excitation: a tool for fast neural control derived from Volvox carteri, Nat. Neurosci. 11, 631-633.

[11] Klapoetke, N. C., Murata, Y., Kim, S. S., Pulver, S. R., Birdsey-Benson, A., Cho, Y. K., Morimoto, T. K., Chuong, A. S., Carpenter, E. J., Tian, Z., Wang, J., Xie, Y., Yan, Z., Zhang, Y., Chow, B. Y., Surek, B., Melkonian, M., Jayaraman, V., Constantine-Paton, M., Wong, G. K., and Boyden, E. S. (2014) Independent optical excitation of distinct neural populations, Nat. Methods 11, 338-346.

[12] Gunaydin, L. A., Yizhar, O., Berndt, A., Sohal, V. S., Deisseroth, K., and Hegemann, P. (2010) Ultrafast optogenetic control, Nat. Neurosci. 13, 387-392.

[13] Berndt, A., Schoenenberger, P., Mattis, J., Tye, K. M., Deisseroth, K., Hegemann, P., and Oertner, T. G. (2011) High-efficiency channelrhodopsins for fast neuronal stimulation at low light levels, Proc. Natl. Acad. Sci. U. S. A. 108, 7595-7600.

[14] Fenno, L., Yizhar, O., and Deisseroth, K. (2011) The development and application of optogenetics, Annu. Rev. Neurosci. 34, 389-412. 
[15] Kleinlogel, S., Feldbauer, K., Dempski, R. E., Fotis, H., Wood, P. G., Bamann, C., and Bamberg, E. (2011) Ultra light-sensitive and fast neuronal activation with the $\mathrm{Ca}^{2+}$ permeable channelrhodopsin CatCh, Nat. Neurosci. 14, 513-518.

[16] Wen, L., Wang, H., Tanimoto, S., Egawa, R., Matsuzaka, Y., Mushiake, H., Ishizuka, T., and Yawo, H. (2010) Opto-current-clamp actuation of cortical neurons using a strategically designed channelrhodopsin, PLoS One 5, e12893.

[17] Lin, J. Y., Lin, M. Z., Steinbach, P., and Tsien, R. Y. (2009) Characterization of engineered channelrhodopsin variants with improved properties and kinetics, Biophys. J. 96, 18031814.

[18] Ganapathy, S., Venselaar, H., Chen, Q., de Groot, H. J., Hellingwerf, K. J., and de Grip, W. J. (2017) Retinal-based proton pumping in the near infrared, J. Am. Chem. Soc. 139, 2338-2344.

[19] Jokela-Määttä, M., Pahlberg, J., Lindström, M., Zak, P. P., Porter, M., Ostrovsky, M. A., Cronin, T. W., and Donner, K. (2005) Visual pigment absorbance and spectral sensitivity of the Mysis relicta species group (Crustacea, Mysida) in different light environments, J. Comp. Physiol. A Neuroethol. Sens. Neural. Behav. Physiol. 191, 1087-1097.

[20] Ala-Laurila, P., Donner, K., Crouch, R. K., and Cornwall, M. C. (2007) Chromophore switch from 11-cis-dehydroretinal (A2) to 11-cis-retinal (A1) decreases dark noise in salamander red rods, J. Physiol. 585, 57-74.

[21] Yokoyama, S., and Radlwimmer, F. B. (2001) The molecular genetics and evolution of red and green color vision in vertebrates, Genetics 158, 1697-1710.

[22] Provencio, I., Loew, E. R., and Foster, R. G. (1992) Vitamin A2-based visual pigments in fully terrestrial vertebrates, Vision Res. 32, 2201-2208.

[23] Dartnall, H. J., and Lythgoe, J. N. (1965) The spectral clustering of visual pigments, Vision Res. 5, 81-100.

[24] Sineshchekov, O. A., Govorunova, E. G., Wang, J., and Spudich, J. L. (2012) Enhancement of the long-wavelength sensitivity of optogenetic microbial rhodopsins by 3,4-dehydroretinal, Biochemistry 51, 4499-4506.

[25] Tokunaga, F., and Ebrey, T. (1978) The blue membrane: the 3-dehydroretinal-based artificial pigment of the purple membrane, Biochemistry 17, 1915-1922.

[26] Spudich, J. L., McCain, D. A., Nakanishi, K., Okabe, M., Shimizu, N., Rodman, H., Honig, B., and Bogomolni, R. A. (1986) Chromophore/protein interaction in bacterial sensory rhodopsin and bacteriorhodopsin, Biophys. J. 49, 479-483.

[27] Lanyi, J. K., Zimányi, L., Nakanishi, K., Derguini, F., Okabe, M., and Honig, B. (1988) Chromophore/protein and chromophore/anion interactions in halorhodopsin, Biophys. J. 53, 185-191. 
[28] Takahashi, T., Yan, B., Mazur, P., Derguini, F., Nakanishi, K., and Spudich, J. L. (1990) Color regulation in the archaebacterial phototaxis receptor phoborhodopsin (sensory rhodopsin II), Biochemistry 29, 8467-8474.

[29] Iwasa, T. (1992) Artificial pigments of halorhodopsin and their chloride pumping activities, Biochemistry 31, 1190-1195.

[30] Smolensky, E., and Sheves, M. (2009) Retinal-salinixanthin interactions in xanthorhodopsin: a circular dichroism (CD) spectroscopy study with artificial pigments, Biochemistry 48, 8179-8188.

[31] Ganapathy, S., Bécheau, O., Venselaar, H., Frölich, S., van der Steen, J. B., Chen, Q., Radwan, S., Lugtenburg, J., Hellingwerf, K. J., de Groot, H. J., and de Grip, W. J. (2015) Modulation of spectral properties and pump activity of proteorhodopsins by retinal analogues, Biochem. J. 467, 333-343.

[32] Okitsu, T., Matsuyama, T., Yamashita, T., Ishizuka, T., Yawo, H., Imamoto, Y., Shichida, Y., and Wada, A. (2017) Alternative formation of red-shifted channelrhodopsins: Noncovalent incorporation with retinal-based enamine-type Schiff bases and mutated channelopsin, Chem. Pharm. Bull. (Tokyo) 65, 356-358.

[33] Zingoni, J., Or, Y. S., Crouch, R. K., Chang, C. H., Govindjee, R., and Ebrey, T. G. (1986) Effect of variation of retinal polyene side-chain length on formation and function of bacteriorhodopsin analogue pigments, Biochemistry 25, 2022-2027.

[34] Tokunaga, F., Govindjee, R., and Ebrey, T. G. (1977) Synthetic pigment analogues of the purple membrane protein, Biophys. J. 19, 191-198.

[35] Gärtner, W., Towner, P., Hopf, H., and Oesterhelt, D. (1983) Removal of methyl groups from retinal controls the activity of bacteriorhodopsin, Biochemistry 22, 2637-2644.

[36] Kato, H. E., Zhang, F., Yizhar, O., Ramakrishnan, C., Nishizawa, T., Hirata, K., Ito, J., Aita, Y., Tsukazaki, T., Hayashi, S., Hegemann, P., Maturana, A. D., Ishitani, R., Deisseroth, K., and Nureki, O. (2012) Crystal structure of the channelrhodopsin lightgated cation channel, Nature 482, 369-374.

[37] Nagel, G., Ollig, D., Fuhrmann, M., Kateriya, S., Musti, A. M., Bamberg, E., and Hegemann, P. (2002) Channelrhodopsin-1: a light-gated proton channel in green algae, Science 296, 2395-2398.

[38] Nagel, G., Szellas, T., Huhn, W., Kateriya, S., Adeishvili, N., Berthold, P., Ollig, D., Hegemann, P., and Bamberg, E. (2003) Channelrhodopsin-2, a directly light-gated cation-selective membrane channel, Proc. Natl. Acad. Sci. U. S. A. 100, 13940-13945.

[39] Takayama, R., Kaneko, A., Okitsu, T., Tsunoda, S. P., Shimono, K., Mizuno, M., Kojima, K., Tsukamoto, T., Kandori, H., Mizutani, Y., Wada, A., and Sudo, Y. (2018) Production of a Light-Gated Proton Channel by Replacing the Retinal Chromophore with Its Synthetic Vinylene Derivative, J Phys Chem Lett, 2857-2862. 
[40] Wada, A., Hiraishi, S., Takamura, N., Date, T., Aoe, K., and Ito, M. (1997) A novel method for a stereoselective synthesis of trisubstituted olefin using tricarbonyliron complex: a highly stereoselective synthesis of (all-E)- and (9Z)-retinoic acids, J. Org. Chem. 62, 4343-4348.

[41] Wang, H., Sugiyama, Y., Hikima, T., Sugano, E., Tomita, H., Takahashi, T., Ishizuka, T., and Yawo, H. (2009) Molecular determinants differentiating photocurrent properties of two channelrhodopsins from chlamydomonas, J. Biol. Chem. 284, 5685-5696.

[42] Niwa, H., Yamamura, K., and Miyazaki, J. (1991) Efficient selection for high-expression transfectants with a novel eukaryotic vector, Gene 108, 193-199.

[43] Imamoto, Y., and Shichida, Y. (2008) Thermal recovery of iodopsin from photobleaching intermediates, Photochem. Photobiol. 84, 941-948.

[44] Kojima, K., Imamoto, Y., Maeda, R., Yamashita, T., and Shichida, Y. (2014) Rod visual pigment optimizes active state to achieve efficient $\mathrm{G}$ protein activation as compared with cone visual pigments, J. Biol. Chem. 289, 5061-5073.

[45] Nakanishi, K., and Crouch, R. (1995) Application of artificial pigments to structure determination and study of photoinduced transformations of retinal proteins, Isr. J. Chem. 35, 253-272.

[46] AzimiHashemi, N., Erbguth, K., Vogt, A., Riemensperger, T., Rauch, E., Woodmansee, D., Nagpal, J., Brauner, M., Sheves, M., Fiala, A., Kattner, L., Trauner, D., Hegemann, P., Gottschalk, A., and Liewald, J. F. (2014) Synthetic retinal analogues modify the spectral and kinetic characteristics of microbial rhodopsin optogenetic tools, Nat. Commun. 5, 5810.

[47] Lórenz-Fonfría, V. A., and Heberle, J. (2014) Channelrhodopsin unchained: structure and mechanism of a light-gated cation channel, Biochim. Biophys. Acta 1837, 626-642.

[48] Hontani, Y., Marazzi, M., Stehfest, K., Mathes, T., van Stokkum, I. H. M., Elstner, M., Hegemann, P., and Kennis, J. T. M. (2017) Reaction dynamics of the chimeric channelrhodopsin C1C2, Sci. Rep. 7, 7217.

[49] Bamann, C., Gueta, R., Kleinlogel, S., Nagel, G., and Bamberg, E. (2010) Structural guidance of the photocycle of channelrhodopsin-2 by an interhelical hydrogen bond, Biochemistry 49, 267-278.

[50] Berndt, A., Yizhar, O., Gunaydin, L. A., Hegemann, P., and Deisseroth, K. (2009) Bistable neural state switches, Nat. Neurosci. 12, 229-234.

[51] Yizhar, O., Fenno, L. E., Prigge, M., Schneider, F., Davidson, T. J., O'Shea, D. J., Sohal, V. S., Goshen, I., Finkelstein, J., Paz, J. T., Stehfest, K., Fudim, R., Ramakrishnan, C., Huguenard, J. R., Hegemann, P., and Deisseroth, K. (2011) Neocortical excitation/inhibition balance in information processing and social dysfunction, Nature 477, 171-178. 
[52] Prigge, M., Schneider, F., Tsunoda, S. P., Shilyansky, C., Wietek, J., Deisseroth, K., and Hegemann, P. (2012) Color-tuned channelrhodopsins for multiwavelength optogenetics, J. Biol. Chem. 287, 31804-31812.

[53] Lórenz-Fonfría, V. A., Resler, T., Krause, N., Nack, M., Gossing, M., Fischer von Mollard, G., Bamann, C., Bamberg, E., Schlesinger, R., and Heberle, J. (2013) Transient protonation changes in channelrhodopsin-2 and their relevance to channel gating, Proc. Natl. Acad. Sci. U. S. A. 110, E1273-1281. 


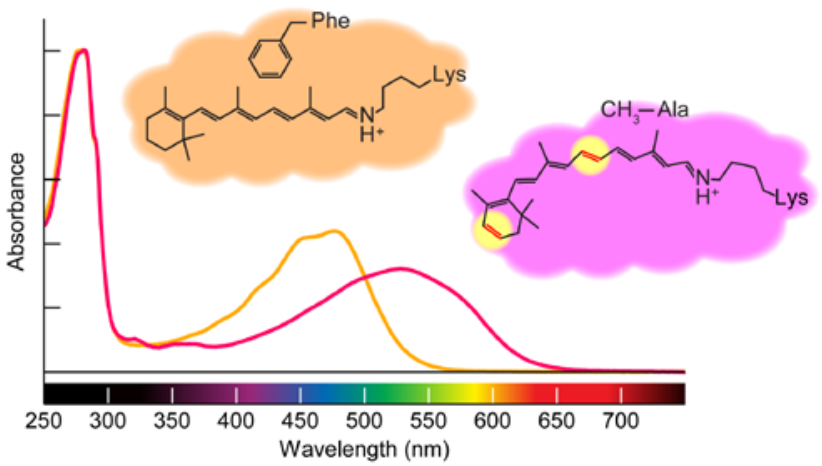

TOC Graphic 


\title{
SUPPORTING INFORMATION
}

\section{Red-Tuning of Channelrhodopsin Spectrum Using Long-Conjugated Retinal Analogues}

\author{
Yi-Chung Shen, ${ }^{\dagger}$ Toshikazu Sasaki, ${ }^{\dagger}$ Take Matsuyama, ${ }^{\dagger}$ Takahiro Yamashita, ${ }^{\dagger}$ Yoshinori Shichida, $^{\dagger, \neq}$ \\ Takashi Okitsu, " Yumiko Yamano," Akimori Wada," Toru Ishizuka, ${ }^{\S}$ Hiromu Yawo, ${ }^{\S}$ and Yasushi \\ Imamoto ${ }^{*}, \dagger$
}

${ }^{\dagger}$ Department of Biophysics, Graduate School of Science, Kyoto University, Kyoto 606-8502, Japan

${ }^{\ddagger}$ Research Organization of Science and Technology, Ritsumeikan University, Kusatsu, Shiga 525-8577, Japan

"Laboratory of Organic Chemistry for Life Science, Kobe Pharmaceutical University, Kobe, Hyogo 658-0003,

Japan

${ }^{\S}$ Department of Developmental Biology and Neuroscience, Graduate School of Life Science, Tohoku University, Sendai, Miyagi 980-8577, Japan

*Corresponding author

Figure S1: Time-resolved UV-visible difference absorption spectra of C1C2 bearing ATR1, ATR2, or their analogues.

Figure S2: $\quad$ SVD-analyzed time-resolved UV-visible absolute spectra of C1C2 bearing ATR1, ATR2, or their analogues.

Figure S3: UV-visible absorption spectra of C1C2 mutants bearing ATR1 retinal and analogues.

Figure S4: Time-resolved UV-visible difference absorption spectra of C1C2-F265A bearing ATR1, ATR2, or their analogues.

Figure S5: $\quad$ SVD-analyzed time-resolved UV-visible absolute spectra of C1C2-F265A bearing ATR1, ATR2, or their analogues.

Table S1: Time constants of transitions in C1C2 photocycle.

Table S2: Time constants of transitions in C1C2-F265A photocycle. 

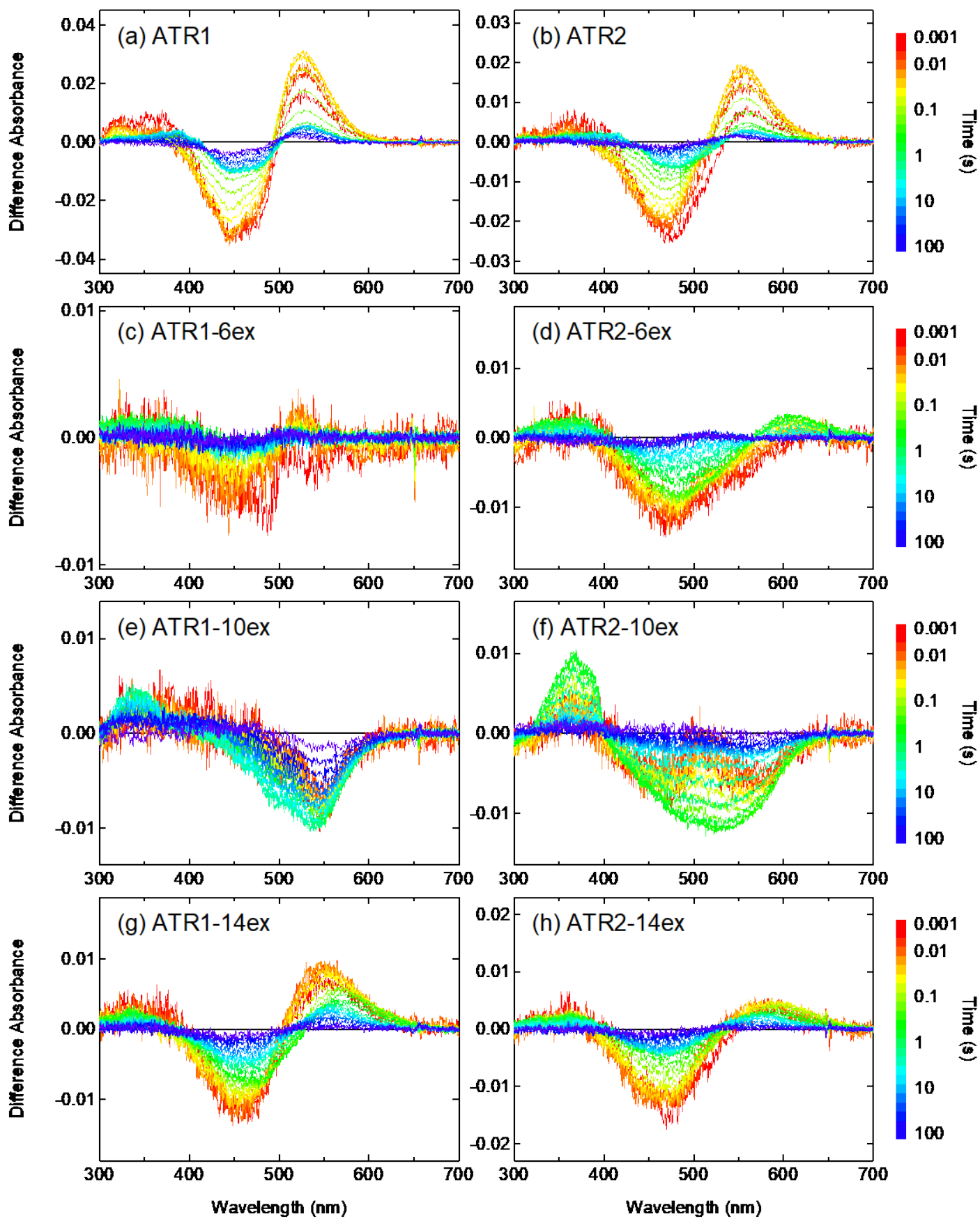

Figure S1. Time-resolved UV-visible difference absorption spectra of C1C2 bearing ATR1, ATR2, or their analogues. The difference spectra before and after photoexcitation with a yellow flash $(>430 \mathrm{~nm}$, pulse duration $=170 \mu \mathrm{s})$ were measured in buffer $(0.02 \%$ DDM, $50 \mathrm{mM}$ HEPES, $140 \mathrm{mM} \mathrm{NaCl}, \mathrm{pH}$ 6.5) at 20 $\mathrm{C}$. (a) ATR1-C1C2. (b) ATR2-C1C2. (c) ATR1-6ex-C1C2. (d) ATR26ex-C1C2. (e) ATR1-10ex-C1C2. (f) ATR2-10ex-C1C2. (g) ATR1-14ex-C1C2. (h) ATR2-14ex-C1C2. 

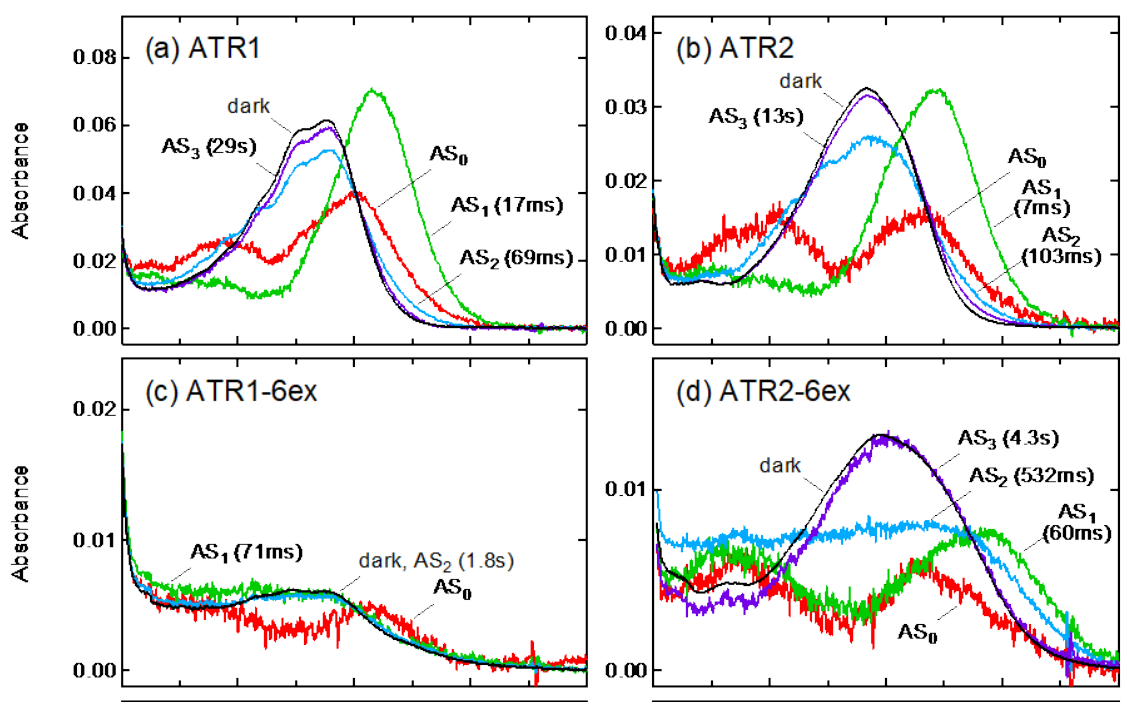

(d) ATR2-6ex
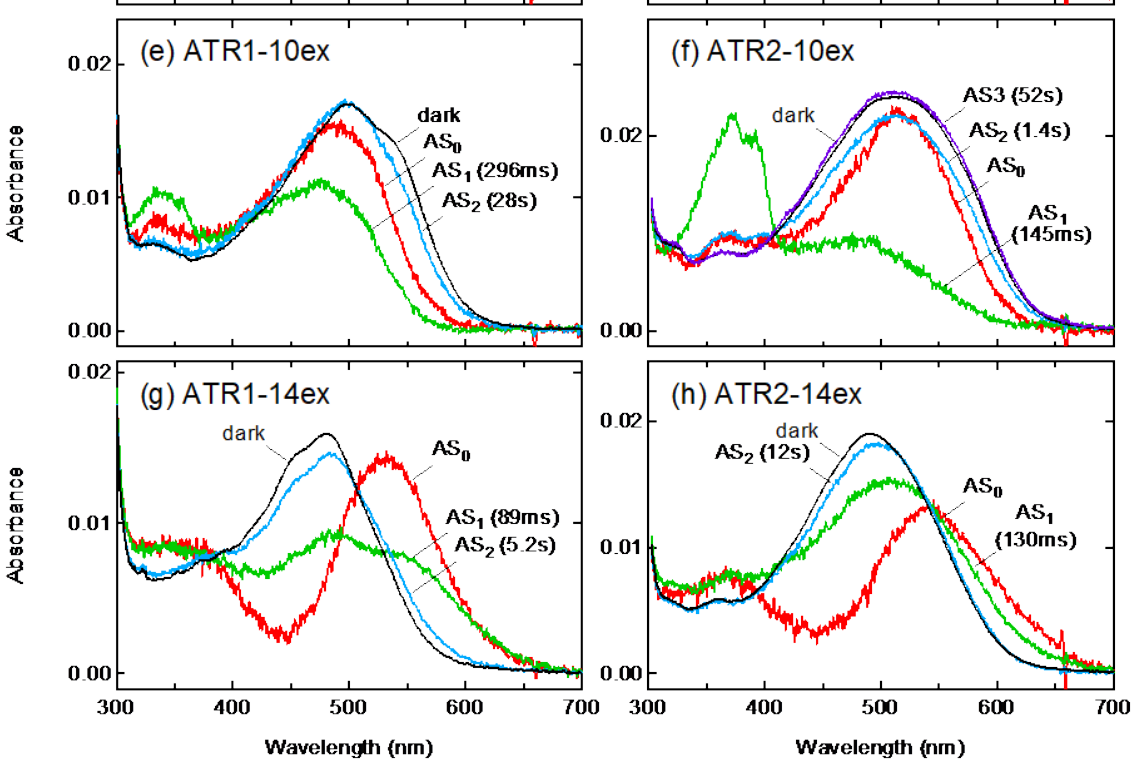

Figure S2. SVD-analyzed time-resolved UV-visible absolute spectra of C1C2 bearing ATR1, ATR2, or their analogues. These spectra were constructed using the B-spectra and dark state spectra measured in buffer (0.02\% DDM, $50 \mathrm{mM}$ HEPES, $140 \mathrm{mM} \mathrm{NaCl}$, pH 6.5) at $20^{\circ} \mathrm{C}$ (see Materials and Methods). (a) ATR1-C1C2. (b) ATR2-C1C2. (c) ATR1-6ex-C1C2. (d) ATR2-6ex-C1C2. (e) ATR1-10ex-C1C2. (f) ATR2-10ex-C1C2. (g) ATR1-14ex (h) ATR2-14ex-C1C2. 

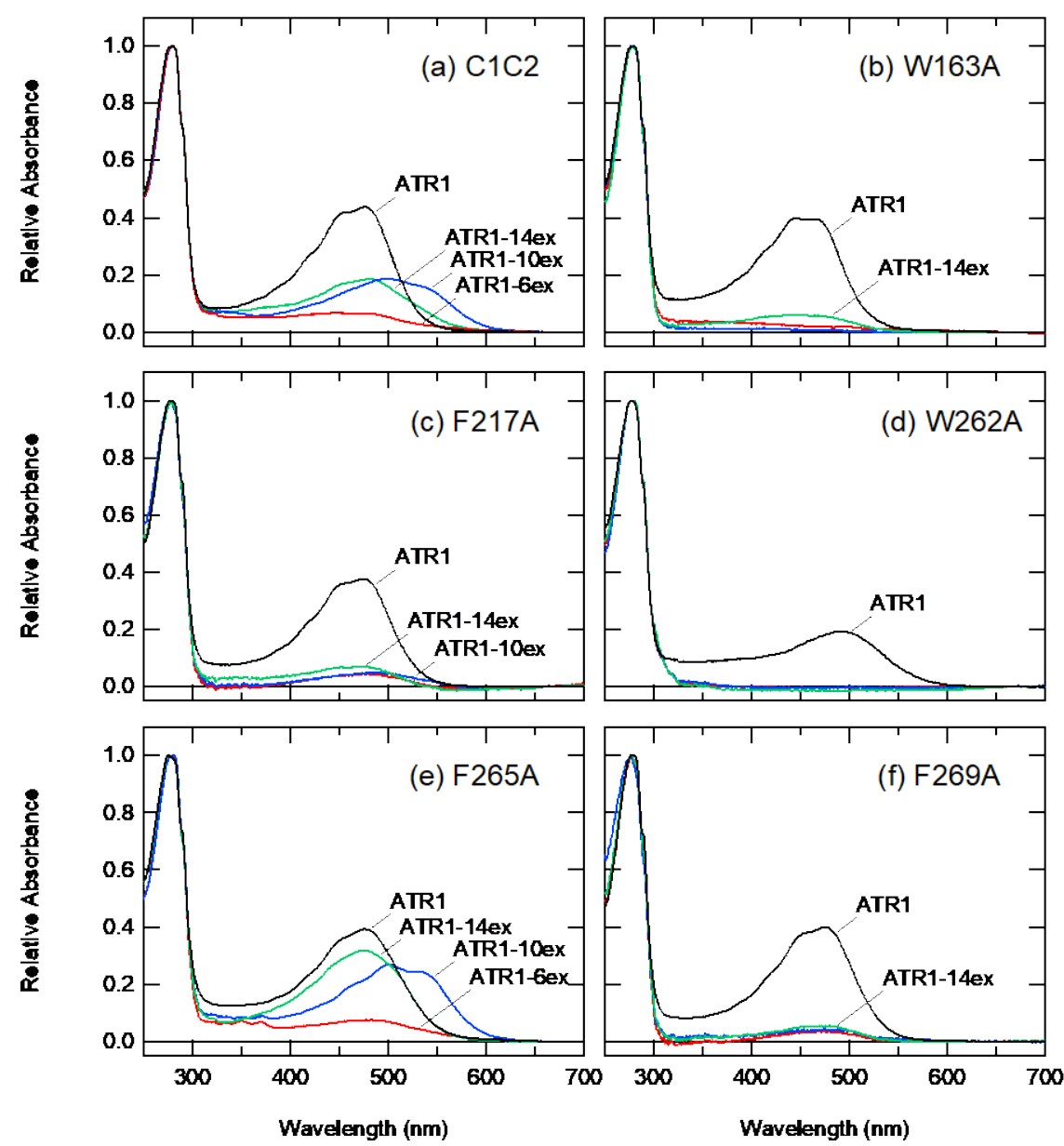

Figure S3. UV-visible absorption spectra of C1C2 mutants bearing ATR1 retinal and analogues. Mutation of aromatic residues in RBP other than Phe265 caused destructive effect to the pigment formation with ATR1- retinal and analogues. All samples were dissolved in buffer (0.02\% DDM, 50 mM HEPES, 140 mM NaCl, pH 6.5). (a) C1C2. (b) W163A. (c) F217A. (d) W262A. (e) F265A. (f) F269A. 

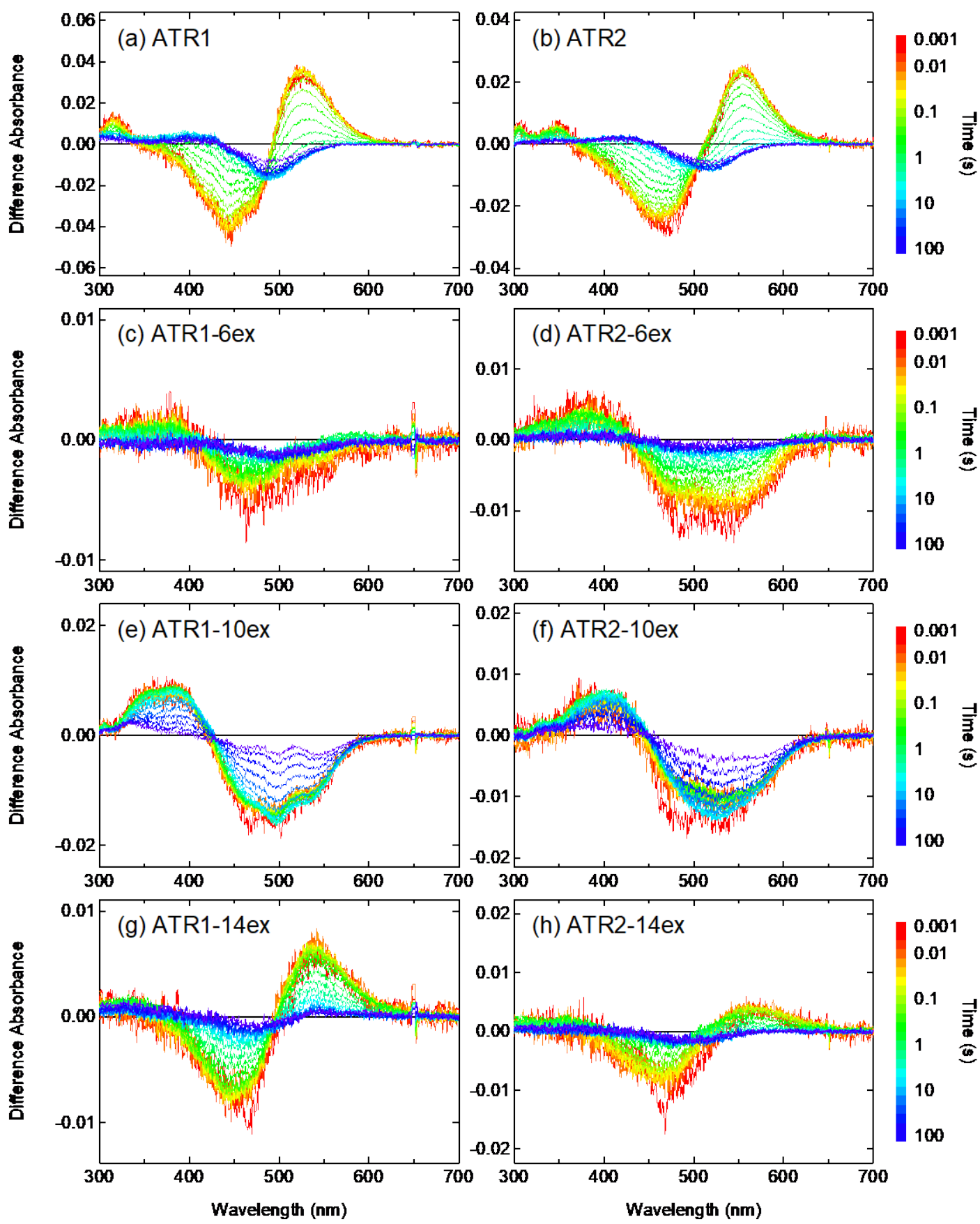

Figure S4. Time-resolved UV-visible difference absorption spectra of F265A bearing ATR1, ATR2, or their analogues. The difference spectra before and after photoexcitation with a yellow flash $(>430$ $\mathrm{nm}$, pulse duration $=170 \mu \mathrm{s})$ were measured in buffer (0.02\% DDM, $50 \mathrm{mM}$ HEPES, $140 \mathrm{mM} \mathrm{NaCl}$, pH 6.5) at $20^{\circ}$ C. (a) ATR1-F265A. (b) ATR2-F265A. (c) ATR1-6ex-F265A. (d) ATR2-6ex-F265A. (e) ATR1-10ex-F265A. (f) ATR2-10ex-F265A. (g) ATR1-14ex-F265A. (h) ATR2-14ex-F265A. 

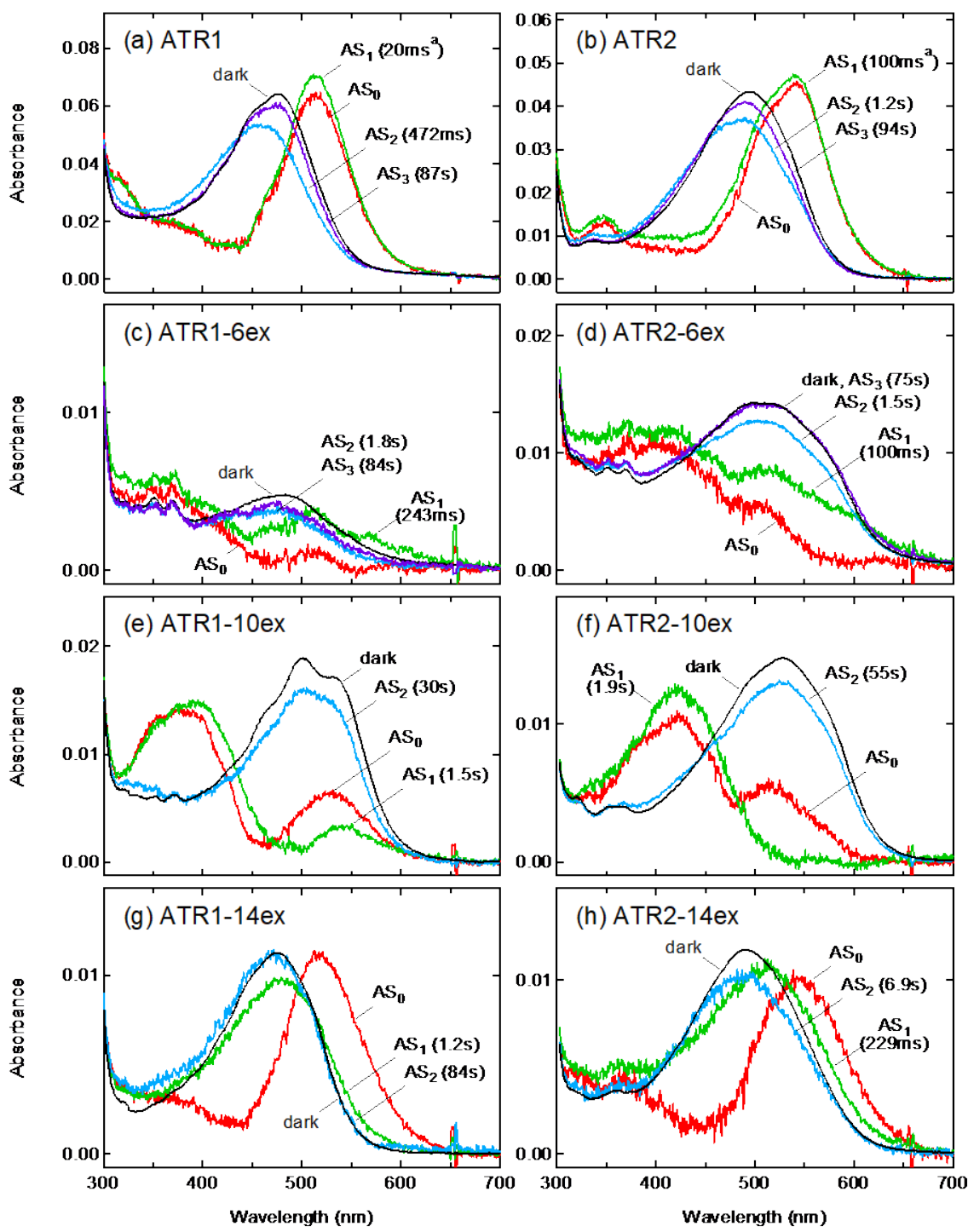

Figure S5. SVD-analyzed time-resolved UV-visible absolute spectra of F265A bearing ATR1, ATR2, or their analogues. These spectra were constructed using the B-spectra and dark state spectra measured in buffer (0.02\% DDM, $50 \mathrm{mM}$ HEPES, $140 \mathrm{mM} \mathrm{NaCl}$, pH 6.5) at $20^{\circ} \mathrm{C}$ (see Materials and Methods). (a) ATR1-F265A. (b) ATR2-F265A. (c) ATR1-6ex-F265A. (d) ATR2-6ex-F265A. (e) ATR110ex-F265A. (f) ATR2-10ex-F265A. (g) ATR1-14ex-F265A. (h) ATR2-14ex-F265A. 
Table S1. Time constants of transitions in C1C2 photocycle

\begin{tabular}{|c|c|c|c|c|c|c|c|c|}
\hline \multirow[b]{3}{*}{ Transition $^{\mathrm{a}}$} & \multicolumn{8}{|c|}{ Time constant (ms) } \\
\hline & \multicolumn{4}{|c|}{ ATR1-type } & \multicolumn{4}{|c|}{ ATR2-type } \\
\hline & ATR1 & $6 e x$ & $10 \mathrm{ex}$ & $14 \mathrm{ex}$ & ATR2 & $6 e x$ & $10 \mathrm{ex}$ & $14 \mathrm{ex}$ \\
\hline Dark $\rightarrow \mathrm{P} 1^{500}$ & - & - & $<2$ & - & - & - & $<2$ & - \\
\hline $\mathrm{P} 1^{500} \rightarrow \mathrm{P} 2^{390}$ & $<2^{\mathrm{b}}$ & - & 296 & - & $<2^{\mathrm{b}}$ & $<2$ & 145 & - \\
\hline $\mathrm{P} 2^{390} \rightarrow \mathrm{P} 3^{520}$ & 16.8 & - & \multirow{2}{*}{\} 27700} & $<2$ & 6.9 & 60.3 & \multirow{2}{*}{\} 1390} & $<2$ \\
\hline $\mathrm{P}^{520} \rightarrow \mathrm{P}^{480}$ & 69.0 & 71.2 & & 88.8 & 103 & 532 & & 130 \\
\hline $\mathrm{P} 4^{480} \rightarrow$ Dark & 28700 & 1800 & - & 5190 & 12900 & 4320 & 51900 & 11500 \\
\hline
\end{tabular}

a Suffixes of the name of intermediates show the absorption maxima in ATR1-C1C2. ${ }^{\text {PP } 3{ }^{520}}$ coexisted.

Table S2. Time constants of transitions in C1C2-F265A photocycle

\begin{tabular}{|c|c|c|c|c|c|c|c|c|}
\hline \multirow[b]{3}{*}{ Transition $^{\mathrm{a}}$} & \multicolumn{8}{|c|}{ Time constant (ms) } \\
\hline & \multicolumn{4}{|c|}{ ATR1-type } & \multicolumn{4}{|c|}{ ATR2-type } \\
\hline & ATR1 & $6 \mathrm{ex}$ & $10 \mathrm{ex}$ & $14 \mathrm{ex}$ & ATR2 & $6 e x$ & $10 \mathrm{ex}$ & $14 \mathrm{ex}$ \\
\hline Dark $\rightarrow \mathrm{P} 1^{500}$ & - & - & $<2$ & - & - & - & $<2$ & - \\
\hline $\mathrm{P} 1^{500} \rightarrow \mathrm{P} 2^{390}$ & $<2^{\mathrm{b}}$ & $<2$ & 1530 & - & $<2^{\mathrm{b}}$ & $<2$ & 1900 & - \\
\hline $\mathrm{P} 2^{390} \rightarrow \mathrm{P}^{520}$ & 20.2 & 243 & \multirow{2}{*}{\} 30200} & $<2$ & 100 & 99.9 & \multirow{2}{*}{\} 54600} & $<2$ \\
\hline $\mathrm{P}^{520} \rightarrow \mathrm{P}^{480}$ & 472 & 1800 & & 1200 & 1190 & 1460 & & 229 \\
\hline $\mathrm{P} 4{ }^{480} \rightarrow$ Dark & 87400 & 84100 & - & 84200 & 94300 & 74900 & - & 6890 \\
\hline
\end{tabular}

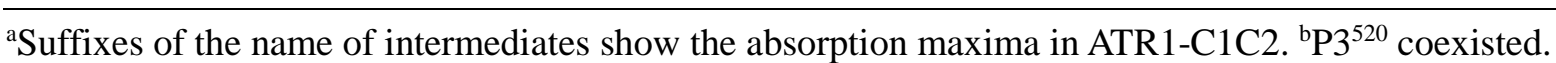

